\title{
Trialitarian automorphisms of lie algebras
}

\section{Journal Article}

Author(s):

Knus, Max-Albert

Publication date:

2009

Permanent link:

https://doi.org/10.3929/ethz-b-000106671

Rights / license:

In Copyright - Non-Commercial Use Permitted

Originally published in:

Transformation groups 14(2), https://doi.org/10.1007/s00031-008-9044-7 


\title{
TRIALITARIAN AUTOMORPHISMS OF LIE ALGEBRAS
}

\author{
M.-A. KNUS \\ ETH Zürich \\ Mathematik Departement \\ CH-8092 Zürich, Switzerland \\ knus@math.ethz.ch
}

\begin{abstract}
Over an algebraically closed field of characteristic zero simple Lie algebras admit outer automorphisms of order 3 if and only if they are of type $\mathrm{D}_{4}$. Moreover, there are two conjugacy classes of such automorphisms. Among orthogonal Lie algebras over arbitrary fields of characteristic zero, only orthogonal Lie algebras relative to quadratic norm forms of Cayley algebras admit outer automorphisms of order 3 . We give a complete list of conjugacy classes of outer automorphisms of order 3 for orthogonal Lie algebras over arbitrary fields of characteristic zero. For the norm form of a given Cayley algebra, one class is associated with the Cayley algebra and the others with central simple algebras of degree 3 with involution of the second kind such that the cohomological invariant of the involution is the norm form.
\end{abstract}

\section{Introduction}

Simple Lie algebras over algebraically closed fields of characteristic zero are classified by their Dynkin diagrams, and the group of automorphisms of the Lie algebra modulo the subgroup of inner automorphisms is isomorphic to the group of symmetries of the corresponding Dynkin diagram. In most cases this group of symmetries has at most two elements. The case of the Lie algebra of skewsymmetric $(8 \times 8)$-matrices is exceptional. The Dynkin diagram is of type $D_{4}$ :

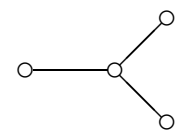

and has the permutation group $S_{3}$ as a group of automorphisms. The existence of peculiar automorphisms of order 3 for Lie algebras of type $D_{4}$ is one aspect of the phenomenon known as triality.

The automorphisms of the Dynkin diagram can easily be extended to automorphisms of the Lie algebra using the root system. Thus over an algebraically closed field, the classes of automorphisms modulo inner automorphisms are explicitly known.

A complete list of conjugacy classes of outer automorphisms of order 3 over an algebraically closed field of characteristic zero can be deduced from the classification of automorphisms of finite order of simple Lie algebras. Such a classification

DOI: $10.1007 / \mathrm{s} 00031-008-9044-7$

Received January 30, 2008. Accepted June 30, 2008. Published online January 3, 2009 
is given for example in [WG], [N], [K69], [K85], [H] or [JZ]. Besides the conjugacy class of the automorphism constructed with the help of the root system, whose fixed point algebra is of type $G_{2}$, there is one more conjugacy class in the full group of automorphisms, whose fixed point algebra is a simple Lie algebra of type $\mathrm{A}_{2}$.

In this work we consider outer automorphisms of order 3 of orthogonal Lie algebras over arbitrary fields of characteristic 0 . The orthogonal Lie algebra relative to the quadratic norm form of a Cayley algebra always admits such automorphisms. This is known as the "local triality principle" (see, e.g., [VBS], [J64], [SV] or Example 7). The following converse also holds: if an orthogonal Lie algebra of type $\mathrm{D}_{4}$ admits an outer automorphism of order 3 , then it is the orthogonal Lie algebra relative to the quadratic norm form of a Cayley algebra. Thus (local) triality and octonions are mutually "responsible" (Tits [T]) for existence. By descent, conjugacy classes of order 3 outer automorphisms must have as fixed point algebras Lie algebras of type $G_{2}$ or $A_{2}$. We show that conjugacy classes of the orthogonal Lie algebra relative to a given norm form are classified by the set consisting of the isomorphism class of the corresponding Cayley algebra (type $\mathrm{G}_{2}$ ) and the isomorphism classes of central simple algebras of degree 3 with specific involutions of the second kind over the quadratic extension generated by a cubic root of unity (type $\mathrm{A}_{2}$ ). We describe an explicit rational construction of a set of representatives of conjugacy classes of outer automorphisms of order 3. For example, over $\mathbb{R}$ there are two conjugacy classes for both the identity norm form and the hyperbolic norm form.

In Section 2 we reproduce the construction of outer automorphisms based on a Cartan decomposition of the Lie algebra. Symmetric compositions and their Lie algebras of derivations are discussed in Sections 3 and 4 . We then recall in Section 5 the construction of outer automorphisms via symmetric compositions given in $[\mathrm{KMRT}]$. In the last section we give a proof that local triality for an orthogonal Lie algebra $\mathfrak{g}$ implies that $\mathfrak{g}$ is the orthogonal Lie algebra of a norm of a Cayley algebra and we show that the classification of symmetric compositions leads to a classification of conjugacy classes of outer automorphisms of order 3 of the Lie algebra. In the Appendix (in collaboration with Larissa Cadorin), we compare the definitions of triality given in Sections 2 and 5 .

If not explicitly mentioned, we assume in the whole paper that fields have characteristic 0 . However, it should be enough to assume characteristic different from 2 and 3.

Acknowledgements. We thank Alberto Elduque and Skip Garibaldi for detecting flaws in preliminary versions, Erhard Neher for showing us a copy of $[\mathrm{N}]$ and telling us about [WG], and O. Loos for useful discussions. The author is also very thankful to the referees for many improvements.

\section{Orthogonal Lie algebras}

Let $V$ be a finite-dimensional vector space over a field $F$ and let $q: V \rightarrow F$ be a quadratic form on $V$, with associated polar form $b_{q}(x, y)=q(x+y)-q(x)-q(y)$. We call the pair $(V, q)$ a quadratic space if $b_{q}$ is nonsingular. The adjoint involution 
$\sigma_{q}$ on $\operatorname{End}_{F}(V)$ is defined through the formula $b_{q}\left(\sigma_{q}(f)(x), y\right)=b_{q}(x, f(y))$ for $x$, $y \in V$ and $f \in \operatorname{End}_{F}(V)$. The space

$$
\begin{aligned}
\mathfrak{o}(V, q)=\mathfrak{o}(q) & =\operatorname{Skew}\left(\operatorname{End}_{F}(V), \sigma_{q}\right) \\
& =\left\{f \in \operatorname{End}_{F}(V) \mid \sigma_{q}(f)=-f\right\} \\
& =\left\{f \in \operatorname{End}_{F}(V) \mid b_{q}(x, f(y))+b_{q}(f(x), y)=0\right\}
\end{aligned}
$$

of skew-symmetric elements of $\operatorname{End}_{F}(V)$ with respect to the involution $\sigma_{q}$ is a Lie subalgebra (of dimension $n(n-1) / 2$ ) of $\operatorname{End}_{F}(V)$ for the Lie bracket $[f, g]=$ $f \circ g-g \circ f$ of $\operatorname{End}_{F}(V)$. The Lie algebra $\mathfrak{o}(q)$ is called the orthogonal Lie algebra relative to $q$.

Example 1. Let $V=F^{2 m}, m \geqslant 4$, and let $b_{2 m}$ be the symmetric bilinear form on $V$ given by the matrix

$$
s=\left(\begin{array}{cc}
0 & I_{m} \\
I_{m} & 0
\end{array}\right)
$$

where $I_{m}$ is the $m$-dimensional identity matrix. The quadratic space $\left(F^{2 m}, b_{2 m}\right)$ is the $2 m$-dimensional hyperbolic space $H_{2 m}$. A basis $\left(e_{1}, \ldots, e_{m}, f_{1}, \ldots, f_{m}\right)$ with

$$
b_{2 m}\left(e_{i}, e_{j}\right)=0, \quad b_{2 m}\left(f_{i}, f_{j}\right)=0, \quad b_{2 m}\left(e_{i}, f_{i}\right)=1, \quad i, j=1, \ldots, m,
$$

is called hyperbolic. The corresponding orthogonal Lie algebra $\mathfrak{o}_{2 m}$ can be identified with the set of matrices

$$
\left(\begin{array}{cc}
X & Y \\
Z & -X^{t}
\end{array}\right)
$$

where $X, Y, Z$ are $(m \times m)$-matrices, $Y$ and $Z$ are skew-symmetric and $X^{t}$ is the transpose of $X$. Orthogonal Lie algebras relative to quadratic spaces of even dimension $2 m$ are forms of $\mathfrak{o}_{2 m}$, i.e., isomorphic to $\mathfrak{o}_{2 m}$ over an algebraic closure $\bar{F}$ of $F$.

A similitude of quadratic spaces $(V, q)$ and $\left(V^{\prime}, q^{\prime}\right)$ is an isometry of $(V, q)$ with $\left(V^{\prime}, \lambda q^{\prime}\right)$ for some $\lambda \in F^{\times}=F \backslash\{0\}$. Any similitude $s$ induces an isomorphism $\operatorname{Int}(s):\left(\operatorname{End}_{F}(V), \sigma_{q}\right) \stackrel{\sim}{\rightarrow}\left(\operatorname{End}_{F}\left(V^{\prime}\right), \sigma_{q^{\prime}}\right)$ of algebras with involutions and a Lie algebra isomorphism $\mathfrak{o}(s): \mathfrak{o}(q) \stackrel{\sim}{\rightarrow} \mathfrak{o}\left(q^{\prime}\right)$. Similitudes of the quadratic space $(V, q)$ are linear automorphisms $f$ of $V$ with $q(f(x))=\mu(f) q(x)$, where $\mu(f) \in$ $F^{\times}$is the multiplier of the similitude. They form a group denoted by $\operatorname{GO}(q)$. If $\operatorname{dim}_{F} V=2 m$ is even, a similitude $f$ is proper if $\operatorname{det}(f)=\mu(f)^{m}$. Proper similitudes form a normal subgroup $\mathrm{GO}^{+}(q)$ of $\mathrm{GO}(q)$ of index 2. Elements of $\mathrm{GO}(q)$ act by inner conjugation as automorphisms of $\left(\operatorname{End}_{F}(V), \sigma_{q}\right)$ and the group of automorphisms of the algebra with involution $\left(\operatorname{End}_{F}(V), \sigma_{q}\right)$ is isomorphic to the projective group $\mathrm{PGO}(q)=\mathrm{GO}(q) / F^{\times}$. The group $\mathrm{PGO}^{+}(q)=\mathrm{GO}^{+}(q) / F^{\times}$ also acts on $\mathfrak{o}(q)$ by conjugations and the corresponding automorphisms are called inner automorphisms of $\mathfrak{o}(q)$. Inner automorphisms can be identified over an algebraically closed field with invariant automorphisms of the Lie algebra $\mathfrak{o}(q)$ (see [J62, Chap. IX]). Inner automorphisms form a normal subgroup $\operatorname{Int}(\mathfrak{o}(q))$ of the full automorphism group $\operatorname{Aut}(\mathfrak{o}(q))$ of $\mathfrak{o}(q)$. Automorphisms which are not inner 
automorphisms are called outer automorphisms. The definitions of inner and outer automorphisms are invariant under base field extensions and are independent of the realization of the Lie algebra in the form $\mathfrak{o}(q)$.

It is a classical result (see, e.g., [J62, Chap. IX] or [SP]) that, over an algebraically closed field of characteristic zero, the factor group

$$
\operatorname{Out}(\mathfrak{o}(q))=\operatorname{Aut}(\mathfrak{o}(q)) / \operatorname{Int}(\mathfrak{o}(q))
$$

is isomorphic to the group of automorphisms of the Dynkin diagram of type $D_{m}$ :

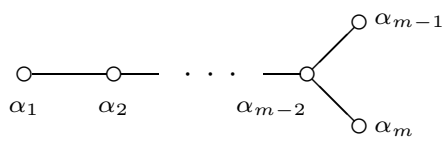

If $m \neq 4$, the Dynkin diagram admits only one nontrivial automorphism, of order 2 (which corresponds to the automorphism of the Lie algebra given by conjugation with a similitude which is not proper). The case of type $D_{4}$ is exceptional, in the sense that the group of automorphisms of the Dynkin diagram is $S_{3}$, the group of permutations of three objects. Thus we have an exact sequence

$$
1 \rightarrow \operatorname{Int}\left(\mathfrak{o}_{8}\right) \rightarrow \operatorname{Aut}\left(\mathfrak{o}_{8}\right) \rightarrow S_{3} \rightarrow 1
$$

Proposition 3. Let $(V, q)$ and $\left(V^{\prime}, q^{\prime}\right)$ be quadratic spaces of even dimension $2 m$, $m \geqslant 4$.

(1) Any similitude $s:(V, q) \stackrel{\sim}{\rightarrow}\left(V^{\prime}, q^{\prime}\right)$ induces an isomorphism

$$
\operatorname{Int}(s):\left(\operatorname{End}_{F}(V), \sigma_{q}\right) \stackrel{\sim}{\rightarrow}\left(\operatorname{End}_{F}\left(V^{\prime}\right), \sigma_{q^{\prime}}\right)
$$

of algebras with involution and conversely any isomorphism

$$
\left(\operatorname{End}_{F}(V), \sigma_{q}\right) \stackrel{\sim}{\rightarrow}\left(\operatorname{End}_{F}\left(V^{\prime}\right), \sigma_{q^{\prime}}\right)
$$

of algebras with involution is of the form $\operatorname{Int}(s)$ for a similitude $s:(V, q) \stackrel{\sim}{\rightarrow}$ $\left(V^{\prime}, q^{\prime}\right)$.

(2) The quadratic spaces $(V, q)$ and $\left(V^{\prime}, q^{\prime}\right)$ are similar if and only if the Lie algebras $\mathfrak{o}(q)$ and $\mathfrak{o}\left(q^{\prime}\right)$ are isomorphic.

Proof. The first claim follows from the fact that any isomorphism of algebras $\operatorname{End}_{F}(V) \stackrel{\sim}{\rightarrow} \operatorname{End}_{F}\left(V^{\prime}\right)$ is induced by an isomorphism $V \stackrel{\sim}{\rightarrow} V^{\prime}$. Claim (2), for $m \neq 4$, is in [J62] and in [J64] for $m=4$.

Following $[\mathrm{N}]$ (see also $[\mathrm{WG}]$ ) we construct in this section outer automorphisms of order 3 of the Lie algebra $\mathfrak{o}_{8}$ using root systems. We fix a Cartan subalgebra $\mathfrak{h}$ of $\mathfrak{o}_{8}$, for example

$$
\mathfrak{h}=\left\{\left(\begin{array}{cc}
D & 0 \\
0 & -D
\end{array}\right) \mid D=\operatorname{diag}\left(\lambda_{1}, \ldots, \lambda_{4}\right), \lambda_{i} \in F\right\} .
$$


Let $\left(E_{i j}, i, j=1, \ldots, n\right)$ be the standard basis of the algebra $M_{n}(F)$ of $(n \times n)$ matrices with entries in $F$. The matrices

$$
u_{j}=E_{j j}-E_{j+4, j+4}, \quad j=1, \ldots, 4,
$$

form a basis of $\mathfrak{h}$. We have a Cartan decomposition of $\mathfrak{o}_{8}$,

$$
\mathfrak{o}_{8}=\mathfrak{h} \oplus\left(\bigoplus_{\alpha} \mathfrak{g}_{\alpha}\right),
$$

where the one-dimensional root spaces $\mathfrak{g}_{\alpha}$ are generated by the 24 matrices

$$
\begin{array}{ll}
G_{i j}^{X}=E_{i, j}-E_{j+4, i+4} & 1 \leqslant i, j \leqslant 4, i \neq j, \\
G_{i j}^{Y}=E_{i, j+4}-E_{j, i+4} & 1 \leqslant i<j \leqslant 4, \\
G_{i j}^{Z}=E_{i+4, j}-E_{j+4, i} & 1 \leqslant i<j \leqslant 4 .
\end{array}
$$

Let $\mathfrak{h}^{*}$ be the dual space of $\mathfrak{H}$ and let $\varepsilon_{i} \in \mathfrak{h}^{*}, i=1, \ldots, 4$, be the dual basis of the basis $\left(u_{j}\right)$ of $\mathfrak{h}$. The simple roots are

$$
\alpha_{1}=\varepsilon_{1}-\varepsilon_{2}, \quad \alpha_{2}=\varepsilon_{2}-\varepsilon_{3}, \quad \alpha_{3}=\varepsilon_{3}-\varepsilon_{4}, \quad \text { and } \quad \alpha_{4}=\varepsilon_{3}+\varepsilon_{4} .
$$

The rule $\alpha_{1} \mapsto \alpha_{4}, \alpha_{4} \mapsto \alpha_{3}, \alpha_{3} \mapsto \alpha_{1}, \alpha_{2} \mapsto \alpha_{2}$ defines an automorphism $\rho$ of the Dynkin diagram $D_{4}$. Let $\bar{\rho}$ be its (unique) extension to $\mathfrak{h}^{*}$. The automorphism $\bar{\rho}$ is given by the matrix

$$
R=\frac{1}{2}\left(\begin{array}{cccc}
1 & 1 & 1 & -1 \\
1 & 1 & -1 & 1 \\
1 & -1 & 1 & 1 \\
1 & -1 & -1 & -1
\end{array}\right)
$$

with respect to the basis $\left(\varepsilon_{i}\right)$. Similarly, let $\pi$ be the automorphism of the Dynkin diagram given by $\alpha_{3} \mapsto \alpha_{4}, \alpha_{4} \mapsto \alpha_{3}, \alpha_{1} \mapsto \alpha_{1}, \alpha_{2} \mapsto \alpha_{2}$. Its extension $\bar{\pi}$ to $\mathfrak{h}^{*}$ has the matrix

$$
P=\frac{1}{2}\left(\begin{array}{cccc}
1 & 0 & 0 & 0 \\
0 & 1 & 0 & 0 \\
0 & 0 & 1 & 0 \\
0 & 0 & 0 & -1
\end{array}\right) .
$$

One checks that $R^{3}=1, P^{2}=1$ and $P R=R^{2} P$. Thus $\{R, P\}$ generates a group isomorphic to $S_{3}$. Let $H \in \mathfrak{h}$ and $e_{\alpha} \neq 0 \in \mathfrak{g}_{\alpha}$. Any automorphism $\phi$ of the root system induces an automorphism $f_{\phi}$ of $\mathfrak{o}_{8}$ :

$$
f_{\phi}\left(H+\sum_{\alpha} s_{\alpha} e_{\alpha}\right)=\left(\phi^{*}\right)^{-1}(H)+\sum_{\alpha} s_{\alpha} e_{\phi(\alpha)}
$$

where $\phi^{*}$ is the dual of $\phi$ and the sum is taken over all roots. In particular, $\rho$ and $\pi$ induce automorphisms $f_{\rho}$ and $f_{\pi}$. Let $\omega$ be a primitive cubic root of 1 . Let $\left(v_{i}\right)$ be the dual basis of $\left(\alpha_{i}\right)$, so that $v_{2}=u_{1}+u_{2}$. The map

$$
f_{2}\left(H+\sum_{\alpha} s_{\alpha} e_{\alpha}\right)=H+\sum_{\alpha} \omega^{\alpha\left(v_{2}\right)} s_{\alpha} e_{\alpha}
$$

is an automorphism of order 3 of $\mathfrak{o}_{8}$ and is invariant, since it is the identity on the Cartan algebra (see [J62]). Hence it is an inner automorphism. Moreover, $f_{\rho}$ and $f_{2}$ commute. Let $f_{\rho}^{\prime}=f_{\rho} \circ f_{2}$. We have (see $[\mathrm{N}]$ or $[\mathrm{WG}]$ ): 
Theorem 6. Assume that $F$ is algebraically closed.

(1) Every outer automorphism of order 3 of $\mathfrak{o}_{8}$ is conjugate in $\operatorname{Aut}\left(\mathfrak{o}_{8}\right)$ to $f_{\rho}$ or $f_{\rho}^{\prime}$ and in $\operatorname{Int}\left(\mathfrak{o}_{8}\right)$ to $f_{\rho}^{ \pm 1}$ or to $f_{\rho}^{\prime \pm 1}$.

(2) The set $\left\{f_{\rho}, f_{\pi}\right\}$ generates a group isomorphic to $S_{3}$.

(3) The fixed point algebra of $f_{\rho}$ is a Lie algebra of type $\mathrm{G}_{2}$ and that of $f_{\rho}^{\prime}$ of type $\mathrm{A}_{2}$.

(4) Fixed point subalgebras under outer automorphisms of order 3 are isomorphic if and only if the automorphisms are conjugate in $\operatorname{Aut}\left(\mathfrak{o}_{8}\right)$.

Example 7. Let $\mathcal{C}$ be a Cayley algebra with norm $n$ and multiplication $x, y \mapsto x y$. The classical local triality principle (see, e.g., [VBS], [SV] or [J64]) states that for every $s \in \mathfrak{o}(n) \subset \operatorname{End}_{F}(\mathcal{C})$ there are unique $t, u \in \mathfrak{o}(n)$ such that

$$
t(x) y+x s(y)=u(x y) \quad \text { for all } x, y \in \mathcal{C} .
$$

Moreover, the map $f: s \mapsto t$ is an automorphism of order 3 and the map $g: s \mapsto u$ is an automorphism of order 2 of $\mathfrak{o}(n)$; they generate a group isomorphic to $S_{3}$. The fixed point Lie algebra $\operatorname{Fix}(f)=\{s \in \mathfrak{o}(n) \mid f(s)=s\}$ is the Lie algebra of derivations of $\mathcal{C}$ which is a Lie algebra of type $\mathrm{G}_{2}$. Thus by Theorem $6 f$ is conjugate to $f_{\rho}$ over an algebraic closure of $F$.

\section{Symmetric compositions}

Let $S$ be a finite-dimensional $F$-vector space with a bilinear multiplication $(x, y) \mapsto x \star y$. We say that a quadratic form $n$ on $S$ is multiplicative if

$$
n(x \star y)=n(x) n(y)
$$

for all $x, y \in S$. A triple $(S, \star, n)$ with a nonsingular multiplicative quadratic form $n$ is called a composition algebra and $n$ is the norm of $S$. By a classical result of Hurwitz (see, e.g., [KMRT, (33.17)]), a complete list of composition algebras with identity is given by the base field $F$, quadratic separable algebras, quaternion algebras and Cayley algebras over $F$. Composition algebras with identity are usually called Hurwitz algebras. If a composition algebra does not have an identity, one can define a new multiplication such that the corresponding algebra is a composition algebra with identity with the same norm (see Kaplansky [KA] or [KMRT, (33.27)]). Thus:

Proposition 9. Composition algebras only occur in dimensions 1, 2, 4 and 8, and their norms are norms of Hurwitz algebras.

Let $\alpha_{1}, \ldots, \alpha_{n} \in F^{\times}$. We denote by $\left\langle\alpha_{1}, \ldots, \alpha_{n}\right\rangle$ the diagonal quadratic form

$$
\left\langle\alpha_{1}, \ldots, \alpha_{n}\right\rangle=\alpha_{1} x_{1}^{2}+\cdots+\alpha_{n} x_{n}^{2}
$$

and by $\left\langle\left\langle\alpha_{1}, \ldots, \alpha_{k}\right\rangle\right\rangle$ the $k$-fold Pfister quadratic form

$$
\left\langle\left\langle\alpha_{1}, \ldots, \alpha_{k}\right\rangle\right\rangle=\left\langle 1, \alpha_{1}\right\rangle \otimes_{F} \cdots \otimes_{F}\left\langle 1, \alpha_{k}\right\rangle .
$$


Norm forms of composition algebras are $k$-Pfister forms for $k=1,2$ and 3 .

We recall that a symmetric bilinear form $b$ on an algebra $(S, \star)$ is called associative or invariant with respect to the multiplication $\star$ of $S$ if

$$
b(x \star y, z)=b(x, y \star z)
$$

holds for all $x, y, z \in S$. If the polar form $b_{n}$ of a composition algebra $S$ is associative, we say that $(S, \star, n)$ is a symmetric composition. Symmetric compositions have the following nice property (see [OO] or [KMRT, (34.1)].

Proposition 10. Let $(S, \star)$ be an algebra and let be $n$ be a nonsingular multiplicative quadratic form on $S$. The following conditions are equivalent:

(1) $(S, \star, n)$ is a symmetric composition.

(2) The norm form $n$ satisfies the relations $x \star(y \star x)=n(x) y=(x \star y) \star x$ for $x, y \in S$.

If $(S, \star)$ is a symmetric composition, the opposite algebra $\left(S^{\mathrm{op}}, \star\right)$ is also a symmetric composition.

Example 11 ((Symmetric compositions of type $\left.\mathrm{G}_{2},[\mathrm{KMRT}]\right)$ or $\left.\left.[\mathrm{EM}]\right)\right)$. Let $\mathcal{C}$ be a Hurwitz algebra with multiplication $(x, y) \mapsto x y$ and norm $n$. The algebra $\mathcal{C}$ admits a conjugation $\pi_{0}: x \mapsto \bar{x}$ which satisfies

$$
\overline{1}=1, \quad \overline{\bar{x}}=x, \quad \overline{x y}=\bar{y} \bar{x} \quad \text { and } \quad b_{n}(x y, z)=b_{n}(x, z \bar{y}) \quad \text { for all } x, y, z \in \mathcal{C} .
$$

The form $n$ is also multiplicative for the multiplication

$$
(x, y) \mapsto x \star y=\bar{x} \bar{y}
$$

and it follows from (12) that $n$ is associative for the $\star$-multiplication. We call $(\mathcal{C}, \star, n)$ a para-Hurwitz algebra. In dimension 8 para-Hurwitz algebras are called para-Cayley algebras (resp., symmetric compositions of type $\mathrm{G}_{2}$ ).

Example 13 ((Symmetric compositions of type $\left.A_{2},[\mathrm{KMRT}]\right)$ or $\left.\left.[\mathrm{EM}]\right)\right)$. Assume that the base field contains a primitive cubic root $\omega$ of 1 . Let $A$ be a central simple algebra of dimension 9 over $F$ (e.g., $A=M_{3}(F)$ ). Let

$$
P_{A, a}(X)=X^{3}-T_{A}(a) X^{2}+S_{A}(a) X^{2}-N_{A}(a)
$$

be the reduced characteristic polynomial of $A$ and let $A^{0}$ be the eight-dimensional space of reduced trace zero elements

$$
A^{0}=\left\{x \in A \mid T_{A}(x)=0\right\} .
$$

We define a multiplication $\star$ on $A^{0}$ by

$$
x \star y=\mu x y+(1-\mu) y x-\frac{1}{3} T_{A}(x y) 1_{A}
$$

where $\mu=(1-\omega) / 3$. The algebra $\left(A^{0}, \star\right)$ is a symmetric composition algebra with norm $n(x)=-\frac{1}{3} S_{A}(x)=\frac{1}{6} T_{A}\left(x^{2}\right)$ (see, e.g., [KMRT, (34.19)]). 
If $F$ does not contain a primitive cubic root of unity $\omega$, one considers central simple algebras $B$ of dimension 9 over the quadratic field extension $K=F(\omega)=$ $F(\sqrt{-3})$, with an involution $\tau$ of the second kind, i.e., $\left.\tau\right|_{K} \neq 1_{K}$. Let $\operatorname{Sym}(B, \tau)$ be the set of symmetric elements in $B$ and let

$$
\operatorname{Sym}(B, \tau)^{0}=\left\{x \in \operatorname{Sym}(B, \tau) \mid T_{B}(x)=0\right\} .
$$

The multiplication defined on $B^{0}$ by (14) induces a product on $\operatorname{Sym}(B, \tau)^{0}$ and with the quadratic form $n(x)=\frac{1}{6} T_{B}\left(x^{2}\right)$, the space $\left(\operatorname{Sym}(B, \tau)^{0}, \star, n\right)$ is a symmetric composition. This type of composition was first considered by Okubo [O] for matrix algebras and by Faulkner $[\mathrm{F}]$ for cubic alternative algebras. They are called Okubo compositions or symmetric compositions of type $\mathrm{A}_{2}$, more precisely of type ${ }^{1} \mathrm{~A}_{2}$ (resp., of type ${ }^{2} \mathrm{~A}_{2}$ ) depending if $F$ contains a primitive cubic root of 1 or not.

It follows from the classification of symmetric compositions (see [EM] or [KMRT, (34.37)]) that in dimensions 1, 2, and 4 para-Hurwitz and Okubo compositions are essentially the same. In dimension 8 we have ([EM] or [KMRT, (34.37)]):

Theorem 15. Let $F$ be a field of characteristic different from 2 and 3 . A symmetric composition $(S, \star, n)$ of dimension 8 is either isomorphic to:

(1) A para-Cayley algebra $(\mathcal{C}, \star)$ for a Cayley algebra $\mathcal{C}$.

(2) A composition algebra of the form $\left(A^{0}, \star\right)$ for a central simple $F$-algebra $A$ of degree 3 if $F$ contains a primitive cubic root of unity.

(3) A composition algebra of the form $\left(\operatorname{Sym}(B, \tau)^{0}, \star, n\right)$ for a central simple $F(\omega)$-algebra $B$ of degree 3 with an involution $\tau$ of the second kind if $F$ does not contain a primitive cubic root of unity $\omega$.

If the quadratic extension $K$ is allowed to be split, i.e., $K \simeq F \times F$, then types ${ }^{1} \mathrm{~A}_{2}$ and ${ }^{2} \mathrm{~A}_{2}$ need not to be distinguished: we replace $A$ of type ${ }^{1} \mathrm{~A}_{2}$ by $B=H(A)=A \times A^{\text {op }}$ and $\tau$ is the twist of the two factors. We say also in this case that $H(A)$ is central simple over $K / F$ and from now on symmetric compositions of type $\mathrm{A}_{2}$ will be represented as $\operatorname{Sym}(B, \tau)^{0}$. The algebras $\mathcal{C}, A$ or $(B, \tau)$ in Theorem 15 are uniquely determined up to isomorphisms by the corresponding symmetric compositions. More precisely,

\section{Proposition 16.}

(1) Any isomorphism $\mathcal{C}_{1} \simeq \mathcal{C}_{2}$ of Cayley algebras is an isomorphism of the associated para-Cayley algebras and conversely.

(2) Let $\left(B_{i}, \tau_{i}\right), i=1,2$, be central simple of degree 3 with involutions of the second kind over $K=F[x] /\left(x^{2}+3\right)$. Any $K$-isomorphism $\left(B_{1}, \tau_{1}\right) \simeq$ $\left(B_{2}, \tau_{2}\right)$ of algebras with involution induces by restriction an isomorphism $\operatorname{Sym}\left(B_{1}, \tau_{1}\right)^{0} \simeq \operatorname{Sym}\left(B_{2}, \tau_{2}\right)^{0}$ of the associated Okubo algebras and conversely.

(3) Any $F$-isomorphism $\left(B_{1}, \tau_{1}\right) \simeq\left(B_{2}, \tau_{2}\right)$ of algebras with involution induces by restriction an isomorphism or an anti-isomorphism $\operatorname{Sym}\left(B_{1}, \tau_{1}\right)^{0} \simeq$ $\operatorname{Sym}\left(B_{2}, \tau_{2}\right)^{0}$ of the associated Okubo algebras and conversely. 
Proof. Claim (1) follows from the fact that isomorphisms of Cayley algebras are compatible with conjugations. We refer to [KMRT, (34.35)] for claim (2). Claim (3) follows from the fact that if $\phi:\left(B_{1}, \tau_{1}\right) \simeq\left(B_{2}, \tau_{2}\right)$ is an $F$-isomorphism which is not $K$-linear, then $\tau_{2} \circ \phi=\phi \circ \tau_{1}$ is a $K$-anti-isomorphism, hence induces an anti-isomorphism of the associated Okubo algebras.

The conjugation $\pi_{0}$ of a Cayley algebra $(\mathcal{C}, n)$ is an $F$-linear involution for the associated para-Cayley algebra $(\mathcal{C}, \star)$ :

$$
\pi_{0}(x \star y)=\pi_{0}(y) \star \pi_{0}(x), \quad \pi_{0}^{2}=1_{\mathcal{C}},
$$

for $x, y \in \mathcal{C}$. The map $\pi_{0}$ is an isometry of $n$, but is improper since $\operatorname{det}\left(\pi_{0}\right)=-1$. For symmetric composition algebras of type $A_{2}$ we have:

Proposition 18. Let $(B, \tau)$ be a central simple $F(\omega)$-algebra $B$ of degree 3 with an involution $\tau$ of the second kind. The following properties are equivalent:

(1) The symmetric composition $S=\left(\operatorname{Sym}(B, \tau)^{0}, \star, n\right)$ admits an F-linear involution $\pi_{0}$.

(2) $S$ is isomorphic to its opposite algebra $S^{\mathrm{op}}$.

(3) $B$ is split.

Proof. Claim(1) clearly implies claim (2). If $\phi$ is an $F$-linear anti-automorphism of $(S, \star)$, then $\phi$ also defines a $K$-anti-automorphism of $B$ since the multiplication of $B$ restricted to $B^{0}$ is given by

$$
x y=(1+\omega) x \star y-\omega y \star x+b_{n}(x, y) \cdot 1
$$

(see [KMRT, §34]. It follows that $B$ has order 2 in the Brauer group of $K$. Since $B$, being of degree 3 , has exponent 1 or $3, B$ is split. If $B$ is split and $x \mapsto \bar{x}, x \in$ $M_{3}(K)$, is elementwise conjugation, then $\bar{\tau}: x \mapsto \overline{\tau(x)}$ is an $F$-linear involution of $B$, hence (1).

\section{Derivations of symmetric compositions}

For any $F$-algebra $A$ (i.e., an $F$-vector space with a bilinear multiplication $(x, y) \mapsto x y)$, the set of $F$-derivations

$$
\operatorname{Der}(A)=\left\{d \in \operatorname{End}_{F}(A) \mid d(x y)=x d(y)+d(x) y\right\}
$$

is a Lie subalgebra of $\operatorname{End}_{F}(A)$. Let $(\mathcal{C}, n)$ be a Cayley algebra with norm $n$ and let

$$
(\mathcal{C}, n)=F \cdot 1 \perp\left(\mathcal{C}^{0}, n^{0}\right)
$$

where $\left(\mathcal{C}^{0}, n^{0}\right)$ is the quadratic subspace of trace 0 elements. The Lie algebra $\operatorname{Der}(\mathcal{C})$ is a subalgebra of $\mathfrak{o}(n)$ (see [J39]) and the eight-dimensional representation $\operatorname{Der}(\mathcal{C}) \subset \operatorname{End}_{F}(\mathcal{C})$ decomposes as the sum of a one-dimensional trivial representation and the seven-dimensional standard representation

$$
\gamma: \operatorname{Der}(\mathcal{C}) \subset \mathfrak{o}\left(n^{0}\right) \subset \operatorname{End}_{F}\left(\mathcal{C}^{0}\right)
$$

which is known to be absolutely irreducible (see [J39]). 
Proposition 20. Let $\phi: \operatorname{Der}(\mathcal{C}) \rightarrow \mathfrak{o}(q)$ be a faithful orthogonal representation for some seven-dimensional quadratic space $(V, q)$. There exists a similitude $s:(V, q) \stackrel{\sim}{\rightarrow}$ $\left(\mathcal{C}^{0}, n^{0}\right)$ such that $\operatorname{Int}(s) \circ \phi=\gamma$.

Proof. The claim is true over an algebraically closed field (see [J39]). Let $t:(V, q) \stackrel{\sim}{\longrightarrow}$ $\left(\mathcal{C}^{0}, n^{0}\right)$ be a similitude over an algebraic closure $\bar{F}$ of $F$ such that $\operatorname{Int}(t) \circ \phi=\gamma$. Since $\gamma(\operatorname{Der}(\mathcal{C}))$ generates $\operatorname{End}_{F}\left(\mathcal{C}^{0}\right)$ (see [J39]), Int $(t)$ is unique, hence by descent restricts to an isomorphism $\psi:\left(\operatorname{End}_{F}(V), \sigma_{q}\right) \stackrel{\sim}{\rightarrow}\left(\operatorname{End}_{F}\left(\mathcal{C}^{0}\right), \sigma_{n^{0}}\right)$ of algebras with involution. By Proposition 3 we have $\psi=\operatorname{Int}(s)$ for a similitude $s:(V, q) \stackrel{\sim}{\rightarrow}$ $\left(\mathcal{C}^{0}, n^{0}\right)$.

Corollary 21. Let $(\mathcal{C}, n)$ and $\left(\mathcal{C}^{\prime}, n^{\prime}\right)$ be Cayley algebras. If the Lie algebra $\operatorname{Der}(\mathcal{C})$ is isomorphic to a subalgebra of $\mathfrak{o}\left(n^{\prime}\right)$, then $\mathcal{C}$ and $\mathcal{C}^{\prime}$ are isomorphic.

Proof. For reasons of dimension the representation $\operatorname{Der}(\mathcal{C}) \subset \mathfrak{o}\left(n^{\prime}\right) \subset \operatorname{End}_{F}\left(\mathcal{C}^{\prime}\right)$ decomposes into a one-dimensional representation $V_{1}$ and a seven-dimensional irreducible representation $V_{2}$. We claim that the decomposition $\mathcal{C}^{\prime}=V_{1} \oplus V_{2}$ is orthogonal with respect to the norm $n^{\prime}$. Let $b^{\prime}$ be the polar of $n^{\prime}$ and let $x_{i} \in V_{i}$, $i=1,2$. The one-dimensional representation is the trivial representation, hence

$$
b^{\prime}\left(d\left(x_{2}\right), x_{1}\right)=-b^{\prime}\left(x_{2}, d\left(x_{1}\right)\right)=0 .
$$

Thus $\operatorname{Der}(\mathcal{C})\left(V_{2}\right)$ is orthogonal to $V_{1}$ and since $\operatorname{Der}(\mathcal{C})$ generates $\operatorname{End}_{F}\left(V_{2}\right)$ as an $F$-algebra, we have an orthogonal decomposition

$$
\left(\mathcal{C}^{\prime}, n^{\prime}\right)=\left(V_{1},\langle\lambda\rangle\right) \perp\left(V_{2}, q\right)
$$

for some quadratic form $q$ on $V_{2}$. By Proposition 20 the form $q$ is isometric to $\langle\mu\rangle \cdot n^{0}$ for some $\mu \in F^{\times}$, thus we get $n^{\prime} \simeq\langle\lambda\rangle \perp\langle\mu\rangle \cdot n^{0}$. Since $\operatorname{det}\left(n^{\prime}\right)$ is congruent to 1 modulo squares in $F,\langle\lambda\rangle \simeq\langle\mu\rangle$ and

$$
n^{\prime} \simeq\langle\mu\rangle \perp\langle\mu\rangle \cdot n^{0} \simeq\langle\mu\rangle \cdot n .
$$

Thus $n$ and $n^{\prime}$ are similar, which implies that $\mathcal{C}$ and $\mathcal{C}^{\prime}$ are isomorphic (see, e.g., [KMRT, (33.19)]).

Corollary 22. Let $(\mathcal{C}, n)$ be a Cayley algebra, let $\gamma: \operatorname{Der}(\mathcal{C}) \rightarrow \mathfrak{o}(n)$ be the standard embedding and let $\mathfrak{g}$ be a Lie subalgebra of $\mathfrak{o}(n)$ isomorphic to $\operatorname{Der}(\mathcal{C})$. Any isomorphism $\phi: \gamma(\operatorname{Der}(\mathcal{C})) \stackrel{\sim}{\rightarrow} \mathfrak{g}$ can be extended to an inner automorphism of $\mathfrak{o}(n)$.

Proof. The isomorphism $\phi$ induces an eight-dimensional representation

$$
\tilde{\phi}: \operatorname{Der}(\mathcal{C}) \stackrel{\sim}{\rightarrow} \mathfrak{g} \subset \mathfrak{o}(n) \subset \operatorname{End}_{F}(\mathcal{C})
$$

which decomposes as a direct sum of a one-dimensional and a seven-dimensional representation

$$
(\mathcal{C}, n)=(F \cdot e,\langle\lambda\rangle) \perp(V, q) .
$$

By Proposition 20 there is an isometry $s:(V, q) \stackrel{\sim}{\rightarrow}\langle\mu\rangle \cdot\left(\mathcal{C}^{0}, n^{0}\right), \mu \in K^{\times}$, such that $\operatorname{Int}(s) \circ \tilde{\phi}=\gamma$. Since $\operatorname{det}(n)$ is congruent to 1 modulo squares, the element $e$ can be chosen such that $n(e)=\mu^{-1}$ and $e \mapsto 1$ extends $s$ to a similitude of $(\mathcal{C}, n)$, also denoted $s$. If $s$ is improper, we may replace $s$ by $\pi_{0} \circ s$, where $\pi_{0}$ is the conjugation of $\mathcal{C}$, since $\operatorname{Int}\left(\pi_{0}\right)$ is the identity on $\operatorname{Der}(\mathcal{C})([\mathrm{S}, \mathrm{III} .8,(3.75)])$. 


\section{Proposition 23.}

(1) Let $(S, \star)$ be the para-Cayley algebra associated with a Cayley algebra $\mathcal{C}$. Then $\operatorname{Der}(S, \star)=\operatorname{Der}(\mathcal{C})$, so that $\operatorname{Der}(S, \star)$ is a simple Lie algebra of type $\mathrm{G}_{2}$.

(2) Let $\left(\mathcal{C}_{i}, n_{i}\right)$ be Cayley algebras with norms $n_{i}$. Any isomorphism $\operatorname{Der}\left(\mathcal{C}_{1}\right) \simeq$ $\operatorname{Der}\left(\mathcal{C}_{2}\right)$ of Lie algebras is induced by a unique isomorphism $\mathcal{C}_{1} \simeq \mathcal{C}_{2}$ of Cayley algebras.

Proof. We refer to [J39] for a proof of 2). To prove 1), first let $d$ be a derivation of $\mathcal{C}, d(x y)=x d(y)+d(x) y$. In view of [S, III.8, (3.75)], we have $d(\bar{x})=\overline{d(x)}$ for $x \in \mathcal{C}$. It follows that

$$
d(x \star y)=d(\bar{x} \bar{y})=\bar{x} d(\bar{y})+d(\bar{x}) \bar{y}=x \star d(y)+d(x) \star y
$$

and $d$ is also a derivation of the associated para-Cayley algebra. Conversely, if $d$ is a derivation of the para-Cayley algebra, one first checks that $d(1)=0$, which implies that $d(x)=-d(\bar{x})$. Using that the conjugation $\pi_{0}: x \mapsto \bar{x}$ is an involution of the para-Cayley algebra, we get

$$
d(x \star y)=d(y) \star \bar{x}+\bar{y} \star d(x) .
$$

With $x=1$ this implies $d(\bar{y})=\overline{d(y)}$ and one concludes that $d$ is also a derivation of the Cayley algebra.

Let $K / F$ be a quadratic extension and let $(B, \tau)$ be a central simple algebra over $K / F$ with an involution $\tau$ of the second kind. The quadratic form $Q: B \rightarrow$ $F, Q(x)=T_{B}(x \tau(x))$ is nonsingular, the involution $\tau$ is an isometry of $Q$ and we have

$$
b_{Q}(x y, z)=b_{Q}(x, z \tau(y))
$$

for $x, y$ and $z \in B$. Let $\operatorname{Skew}(B, \tau)^{0}=[\operatorname{Skew}(B, \tau)$, Skew $(B, \tau)]$ be the Lie algebra of skew-symmetric elements of $B$ of reduced trace 0 . The quadratic form $Q: B \rightarrow$ $F, Q(x)=T_{B}(x \tau(x))$ restricts to a nonsingular quadratic form on $\operatorname{Skew}(B, \tau)^{0}$ denoted by $Q_{-}$and to a nonsingular quadratic form on $\operatorname{Sym}(B, \tau)^{0}$ denoted by $Q_{+}$.

Proposition 24. The map ad: $\operatorname{Skew}(B, \tau)^{0} \rightarrow \operatorname{End}_{F}\left(\operatorname{Skew}(B, \tau)^{0}\right), \operatorname{ad}_{y}(x)=$ $[x, y]$, induces an orthogonal representation

$$
\operatorname{Skew}(B, \tau)^{0} \rightarrow \mathfrak{o}\left(Q_{-}\right)
$$

which is absolutely irreducible.

Proof. The map ad induces an isomorphism

$$
\operatorname{Skew}(B, \tau)^{0} \stackrel{\sim}{\rightarrow} \operatorname{Der}\left(\operatorname{Skew}(B, \tau)^{0}\right)
$$

of Lie algebras since $\operatorname{Skew}(B, \tau)^{0}$ is simple and any derivation of $\operatorname{Skew}(B, \tau)^{0}$ is inner. Using again that any derivation of $\operatorname{Skew}(B, \tau)^{0}$ is inner, it is easy to check that $\operatorname{Der}\left(\operatorname{Skew}(B, \tau)^{0}\right) \subset \mathfrak{o}\left(Q_{-}\right)$. Thus the adjoint representation is orthogonal. The fact that it is absolutely irreducible is for example in [J62]. 
Proposition 26. Let $(B, \tau)$ be a central simple algebra of rank 9 over $K / F$ with an involution of the second kind $\tau$.

(1) Let $(V, q)$ be a eight-dimensional quadratic space and let $\phi: \operatorname{Skew}(B, \tau)^{0} \rightarrow$ $\mathfrak{o}(q)$ be a faithful orthogonal representation. There exists a similitude

$$
s:(V, q) \stackrel{\sim}{\rightarrow}\left(\operatorname{Skew}(B, \tau)^{0}, Q_{-}\right)
$$

such that $\operatorname{Int}(s) \circ \phi=$ ad.

(2) Let $\mathfrak{g}$ be a Lie subalgebra of $\mathfrak{o}\left(Q_{-}\right)$isomorphic to $\operatorname{Der}\left(\operatorname{Skew}(B, \tau)^{0}\right)$. Any isomorphism of Lie algebras $\phi: \operatorname{Der}\left(\operatorname{Skew}(B, \tau)^{0}\right) \stackrel{\sim}{\rightarrow} \mathfrak{g}$ can be extended to an automorphism of $\mathfrak{o}\left(Q_{-}\right)$of the form Int $(s)$, for a similitude $s$ of $Q_{-}$.

Proof. (1) Over an algebraic closure $\bar{F}$ of $F$, the adjoint representation is up to isomorphism the unique irreducible eight-dimensional orthogonal representation of $\operatorname{Skew}(B, \tau)^{0}$. Thus the map $\phi$ can be extended to an isomorphism $\psi=\operatorname{Int}(t)$ of $\left(\operatorname{End}_{F}(V), \sigma_{q}\right) \otimes_{F} \bar{F}$ onto $\left(\operatorname{End}_{F}\left(\operatorname{Skew}(B, \tau)^{0}\right), \sigma_{Q_{-}}\right) \otimes_{F} \bar{F}$, which is uniquely determined by the condition Int $(t) \circ \phi=\mathrm{ad}$, since the image of the map ad in $\operatorname{End}_{\bar{F}}\left(\operatorname{Skew}(B, \tau)^{0}\right)$ generates $\operatorname{End}_{\bar{F}}\left(\operatorname{Skew}(B, \tau)^{0}\right)$. By descent $\psi$ restricts to an isomorphism of $\left(\operatorname{End}_{F}(V), \sigma_{q}\right)$ onto $\left(\operatorname{End}_{F}\left(\operatorname{Skew}(B, \tau)^{0}\right), \sigma_{Q_{-}}\right)$, hence is induced by a similitude of $(V, q)$ with $\left(\operatorname{Skew}(B, \tau)^{0}, Q_{-}\right)$by Proposition 3. Claim (2) follows from claim (1).

Proposition 27. Let $K=F[x] /\left(x^{2}+3\right)$ and let $(B, \tau)$ be central simple of rank 9 over $K / F$, with an involution of the second kind $\tau$. Let $(S, \star, n)=\operatorname{Sym}(B, \tau)^{0}$ be the symmetric composition of type $\mathrm{A}_{2}$ associated with $(B, \tau)$. Let $u$ be a skewsymmetric nonzero element of $K$.

(1) The map $\ell_{u}: x \mapsto u x$ induces a similitude

$$
\left(\operatorname{Sym}(B, \tau)^{0}, n\right) \stackrel{\sim}{\rightarrow}\left(\operatorname{Skew}(B, \tau)^{0}, Q_{-}\right)
$$

and an isomorphism

$$
\operatorname{Int}\left(\ell_{u}\right): \operatorname{Der}(S, \star) \stackrel{\sim}{\rightarrow} \operatorname{Der}(\operatorname{Skew}(B, \tau))
$$

of Lie algebras. Hence the Lie algebra $\operatorname{Der}(S, \star)$ is a simple Lie algebra of type $\mathrm{A}_{2}$.

(2) Let $\left(B_{i}, \tau_{i}\right)$ be central simple algebras with involutions of the second kind over $K / F, i=1,2$. Any isomorphism of Lie algebras $\operatorname{Skew}\left(B_{1}, \tau_{1}\right)^{0} \stackrel{\sim}{\longrightarrow}$ $\operatorname{Skew}\left(B_{2}, \tau_{2}\right)^{0}$ can be uniquely extended to an F-isomorphism $\left(B_{1}, \tau_{1}\right) \stackrel{\sim}{\longrightarrow}$ $\left(B_{2}, \tau_{2}\right)$ and to a K-isomorphism or a K-anti-isomorphism $\left(B_{1}, \tau_{1}\right) \stackrel{\sim}{\rightarrow}$ $\left(B_{2}, \tau_{2}\right)$.

Proof. The first claim of (1) is clear since $n=\frac{1}{6} Q_{+}$and since $Q_{+}$and $Q_{-}$are similar. The second claim follows by a computation using (14) and (17). The fact that $\operatorname{Der}(S, \star, n)$ is a Lie algebra of type $\mathrm{A}_{2}$ is a consequence of (29) and (25). For a proof of (2), see for example [J62]. 
Corollary 30. Let $(B, \tau)$ be a central simple algebra of degree 3 over a quadratic extension $K$ of $F$, with an involution of the second kind. The Lie algebra $\operatorname{Skew}(B, \tau)^{0}$ is a subalgebra of the orthogonal Lie algebra of a 3-Pfister form $n$ if and only if $K=F[x] /\left(x^{2}+3\right)$.

Proof. Assume that $\operatorname{Skew}(B, \tau)^{0}$ is a subalgebra of the orthogonal Lie algebra of a 3-Pfister form $n$. In view of Proposition 26 the form $Q_{-}$is similar to $n$. It is also similar to $\left(\operatorname{Sym}(B, \tau)^{0}, Q_{+}\right)$and, if $K=F[x] /\left(x^{2}-\alpha\right)$,

$$
\left(\operatorname{Sym}(B, \tau)^{0}, Q_{+}\right) \simeq\langle 2,6\rangle \perp\langle 2\rangle \cdot\langle\langle-\alpha\rangle\rangle \cdot q_{0},
$$

where $q_{0}$ is a quadratic space over $F$ of dimension 3 and determinant 1 (see [HKRT] or [KMRT, §19]). Thus the determinant of $Q_{-}$is congruent to $-3 \alpha$ modulo squares. Since the determinant of a 3 -Pfister form is congruent to $1, \alpha$ must be congruent to -3 . The converse follows from Proposition 24 .

By Proposition 9, the norm form $n$ of a symmetric composition of type $\mathrm{A}_{2}$ is a 3-Pfister form. More precisely:

Proposition 32. The norm of a symmetric composition of type $\mathrm{A}_{2}$ is of the form $\langle\langle 3, b, c\rangle\rangle$ for some $b, c \in F^{\times}$. If $F$ contains a primitive cubic root of $1, n$ is hyperbolic.

Proof. By Corollary 30 the element $\alpha$ in (31) is congruent to -3 and, if we write $q_{0}$ in $(31)$ as $\langle b, c, b c\rangle$, we get

$$
n \simeq\left\langle\frac{1}{6}\right\rangle \cdot Q_{+} \simeq\left\langle 1, \frac{1}{3}\right\rangle \perp\left\langle 1, \frac{1}{3}\right\rangle \cdot\langle b, c, b c\rangle \simeq\langle\langle 3, b, c\rangle\rangle
$$

as claimed.

Remark 33. The isomorphism class of the 3-Pfister form given by the norm of the symmetric composition $\operatorname{Sym}(B, \tau)^{0}$ defines a cohomological invariant which classifies involutions of the second kind on $B$. For details see [HKRT] or [KMRT, (19.6)], where this invariant is denoted by $\pi(\tau)$. Those involutions for which the invariant is trivial (i.e., the form is hyperbolic) are called distinguished. Any central simple algebra of degree 3 with involution of the second kind admits a distinguished involution (see [HKRT] or [KMRT, (19.30)]). If $B$ is a matrix algebra and $\tau$ is the Hermitian transpose composed with conjugation by the Hermitian matrix $\left\langle\delta_{1}, \delta_{2}, \delta_{3}\right\rangle, \delta_{i} \in F^{\times}$, then $\pi(\tau) \simeq\left\langle\left\langle 3, \delta_{1} \delta_{2}, \delta_{1} \delta_{3}\right\rangle\right\rangle$.

Example 34. If $F=\mathbb{R}$ and $B=M_{3}(\mathbb{C})$, there are two types of involutions of the second kind on $B$ : the Hermitian transpose, for which the cohomological invariant is the identity form and the Hermitian transpose conjugated with $\langle-1,1,1\rangle$, which is distinguished. Thus there are four classes of symmetric compositions over $\mathbb{R}$.

Proposition 35. Two symmetric compositions of dimension 8 are isomorphic or anti-isomorphic if and only if their Lie algebras of derivations are isomorphic.

Proof. Derivations of $S$ are obviously also derivations of $S^{\text {op }}$. Conversely, in view of Theorem 15 of symmetric compositions, it suffices to check the claim for symmetric compositions $S$ of types $\mathrm{G}_{2}$ and $\mathrm{A}_{2}$. The claim then follows from Corollary 21 and Proposition 27. 


\section{The Clifford algebra of a symmetric composition}

Let $(V, q)$ be a quadratic space and let $C(V, q)$ be the Clifford algebra of the quadratic space $(V, q)$. We recall that $C(V, q)=T V / I$ where $T V$ is the tensor algebra of $V$ and $I$ is the ideal of $T V$ generated by the elements $x \otimes x-q(x) \cdot 1$, $x \in V$. The space $V$, identified with a subspace of $C(V, q)$ through the natural map $V \rightarrow C(V, q)$, generates $C(V, q)$ as an algebra. The even Clifford algebra $C_{0}(V, q)$ is the subalgebra of $C(V, q)$ generated by products of an even number of elements of $V$. The Clifford algebra is characterized by a universal property: any $F$-linear map $\alpha$ of $(V, q)$ to an associative $F$-algebra $A$ such that $\alpha(x)^{2}=q(x)$ for all $x \in V$ factorizes over $C(V, q)$. There is a unique $F$-linear involution $\tau$ on $C(V, q)$ which is the identity on $V$. The structure of the Clifford algebra is described in the following proposition (see, e.g., $[\mathrm{SCH}]$ for a proof).

Proposition 36. Let $(V, q)$ be a nonsingular quadratic space of even dimension $n=2 m$.

(1) The F-algebra $C(V, q)$ is central simple of dimension $2^{n}$.

(2) The center $Z$ of the even Clifford algebra is an étale quadratic extension of $F$ of the form $Z=F(\sqrt{\delta})$ where $\delta=(-1)^{m} \operatorname{det}\left(b_{q}\right)$. The algebra $C_{0}(V, q)$ is central separable over $Z$ of rank $2^{2 m-1}$. Suppose that $m$ is even. Then the involution $\tau$ restricts to an involution $\tau_{0}$ of $C_{0}(V, q)$ which is the identity on $Z$. Further, $\tau_{0}$ is of orthogonal type if $m$ is congruent to 0 modulo 4 and is of symplectic type if $m$ is congruent to 2 modulo 4.

The Lie algebra $\mathfrak{o}(q)$ can be identified with a Lie subalgebra of $\operatorname{Skew}\left(C_{0}(V, q), \tau_{0}\right)$, as follows. For $x, y, z \in V$ and the Lie product $[x, y]=x y-y x$ in $C(V, q)$ we have

$$
[[x, y], z]=2\left(b_{q}(y, z) x-b_{q}(x, z) y\right) \in V .
$$

Let $[V, V]$ be the subspace of $C_{0}(V, q)$ spanned by the $[x, y]=x y-y x$ for all $x$, $y \in V$. In view of (37) we have a linear map

$$
\text { ad: }[V, V] \rightarrow \operatorname{End}_{F}(V), \quad \xi \mapsto \operatorname{ad}_{\xi},
$$

defined by $\operatorname{ad}_{\xi}(z)=[\xi, z]$ for $\xi \in[V, V]$ and $z \in V$.

Lemma 38. The subspace $[V, V]$ of $C_{0}(q)$ is a Lie subalgebra of $\operatorname{Skew}\left(C_{0}(V, q), \tau_{0}\right)$ and ad induces an isomorphism of Lie algebras:

$$
\text { ad: }[V, V] \stackrel{\sim}{\rightarrow} \mathfrak{o}(q) .
$$

Proof. See [KMRT, Lemma (45.3)].

It readily follows from the definition of Clifford algebras that isometries of $(V, q)$ induce automorphisms of $C(V, q)$. For similitudes we have:

Proposition 39. Any similitude $f \in \mathrm{GO}(q)$ with multiplier $m(f)$ induces an automorphism $C(f)$ of $\left(C_{0}(V, q), \tau_{0}\right)$ such that $C(f)(x y)=m(f)^{-1} f(x) f(y)$ for $x$, $y \in V$. The automorphism $C(f)$ restricts to the identity on the center $Z$ of $C_{0}(V, q)$ if and only if $f$ is proper. Further the automorphism ad $\circ C(f) \circ \mathrm{ad}^{-1}$ of $\mathfrak{o}(q)$ is equal to the restriction of $\operatorname{Int}(f)$ to $\mathfrak{o}(q)$ in $\operatorname{End}_{F}(V)$. 
Proof. The first two claims are standard (see, e.g., [KMRT]). Using (37) we have, for $x, y, z \in V$,

$$
(\operatorname{ad} \circ C(f))([x, y])(z)=2 \mu(f)^{-1}\left(\left(f(x) b_{q}(f(y), z)-f(y) b_{q}(f(x), z)\right)\right.
$$

and

$$
\begin{aligned}
(\operatorname{Int}(f) \circ \mathrm{ad})([x, y])(z) & =2 f\left(x b_{q}\left(y, f^{-1}(z)\right)-y b_{q}\left(x, f^{-1}(z)\right)\right) \\
& =2\left(f(x) b_{q}\left(y, f^{-1}(z)\right)-f(y) b_{q}\left(x, f^{-1}(z)\right)\right) .
\end{aligned}
$$

The last claim follows from $\mu(f)^{-1} b_{q}(f(y), z)=b_{q}\left(y, f^{-1}(z)\right)$.

Let $(S, \star, n)$ be a symmetric composition of dimension 8 . Let $C(S, n)$ be the Clifford algebra and $C_{0}(S, n)$ the even Clifford algebra of $(S, n)$. Let $\tau$ be the involution of $C(S, n)$ which is the identity on $S$. Let $r_{x}(y)=y \star x$ and $\ell_{x}(y)=x \star y$ for $x, y \in S$.

Proposition $40(([\mathrm{KMRT}, 35.1]))$. The map $S \rightarrow \operatorname{End}_{F}(S \oplus S)$ given by

$$
x \mapsto\left(\begin{array}{cc}
0 & \ell_{x} \\
r_{x} & 0
\end{array}\right)
$$

induces isomorphisms

$$
\alpha_{S}:(C(S, n), \tau) \stackrel{\sim}{\rightarrow}\left(\operatorname{End}_{F}(S \oplus S), \sigma_{n \perp n}\right)
$$

and

$$
\alpha_{S}:\left(C_{0}(S, n), \tau\right) \stackrel{\sim}{\rightarrow}\left(\operatorname{End}_{F}(S), \sigma_{n}\right) \times\left(\operatorname{End}_{F}(S), \sigma_{n}\right)
$$

of algebras with involution. Further $\alpha_{S}$ maps $[S, S] \subset C_{0}(S, n)$ to $\mathfrak{o}(n) \times \mathfrak{o}(n)$.

Proof. We have $r_{x} \circ \ell_{x}(y)=\ell_{x} \circ r_{x}(y)=n(x) \cdot y$ by Proposition 10 . Thus the existence of the map $\alpha_{S}$ follows from the universal property of the Clifford algebra. We refer to [KMRT, (35.1)] for a proof of the other claims.

We get an (injective) homomorphism

$$
\alpha_{S} \circ \operatorname{ad}^{-1}: \mathfrak{o}(n) \rightarrow \mathfrak{o}(n) \times \mathfrak{o}(n) \subset \operatorname{End}_{F}(S) \times \operatorname{End}_{F}(S)
$$

For any $\lambda \in \mathfrak{o}(n)$, let

$$
\alpha_{S} \circ \operatorname{ad}^{-1}(\lambda)=\left(\lambda^{+}, \lambda^{-}\right)
$$

Theorem 43. For any $\lambda \in \mathfrak{o}(n)$, the elements $\lambda^{+}, \lambda^{-} \in \mathfrak{o}(n)$ satisfy

$$
\begin{aligned}
\lambda^{+}(x \star y) & =\lambda(x) \star y+x \star \lambda^{-}(y), \\
\lambda^{-}(x \star y) & =\lambda^{+}(x) \star y+x \star \lambda(y), \\
\lambda(x \star y) & =\lambda^{-}(x) \star y+x \star \lambda^{+}(y),
\end{aligned}
$$

for all $x, y \in \mathfrak{o}(n)$. Any of the above three relations determines the other two. Further, the pair $\left(\lambda^{+}, \lambda^{-}\right)$is uniquely determined by any of the three relations. 
Proof. See [KMRT, §45].

Let $\rho_{S}$ (resp., $\rho_{S}^{\prime}$ ) be the endomorphisms of $\mathfrak{o}(n)$ defined by $\rho_{S}(\lambda)=\lambda^{+}$and $\rho_{S}^{\prime}(\lambda)=\lambda^{-}$for $\lambda \in \mathfrak{o}(n)$, so that $\alpha_{S} \circ \operatorname{ad}^{-1}=\left(\rho_{S}, \rho_{S}^{\prime}\right) \in \operatorname{End}_{F}(\mathfrak{o}(n)) \times \operatorname{End}_{F}(\mathfrak{o}(n))$. Theorem 43 leads to a description of local triality which is more symmetric than the description given in Example 7.

Theorem 44. The endomorphisms $\rho_{S}$ and $\rho_{S}^{\prime}$ are outer automorphisms of $\mathfrak{o}(n)$ and satisfy the relations $\rho_{S}^{2}=\rho_{S}^{\prime}, \rho_{S}^{3}=1$. Hence $\rho_{S}$ generates a cyclic subgroup of order 3 of $\operatorname{Aut}_{F}(\mathfrak{o}(n))$. The Lie algebra of fixed points under $\rho_{S}$ is the Lie algebra of derivations of $(S, \star)$. Moreover, if $S^{\mathrm{op}}$ is the opposite symmetric composition, then $\rho_{S_{\mathrm{op}}}=\rho_{S}^{2}$, so that $\rho_{S}$ and $\rho_{S^{\mathrm{op}}}$ generate the same cyclic subgroup of order 3 of $\operatorname{Aut}_{F}(\mathfrak{o}(n))$.

Proof. The fact that $\rho_{S}^{2}=\rho_{S}^{\prime}$ and that $\rho_{S}$ generates a cyclic subgroup of order 3 of $\operatorname{Aut}_{F}(\mathfrak{o}(n))$ follows from Theorem 43. The automorphism $\rho_{S}$ is not inner since it permutes the three eight-dimensional representations of $\mathfrak{o}(n)$ (the vector and the two spin-representations). Another proof follows from the direct computations given in the Appendix. The last claim readily follows from the definition of the isomorphism $\alpha_{S}$.

In view of Corollary 21 and Proposition 27 the fixed point Lie algebra of $\rho_{S}$ is a simple Lie algebra of type $\mathrm{G}_{2}$ if $S$ is of type $\mathrm{G}_{2}$ and of type $\mathrm{A}_{2}$ if $S$ is of type $\mathrm{A}_{2}$. Thus the automorphism $\rho_{S}$ is conjugate over an algebraic closure of $F$ (with respect to the full group of automorphisms of $\mathfrak{o}(n))$ to the automorphism $f_{\rho}$ of Theorem 6 if $S$ is a Cayley algebra and to $f_{\rho}^{\prime}$ of Theorem 6 in the other case. If the symmetric composition $S$ is a para-Cayley algebra or is of type $\mathrm{A}_{2}$ for a split algebra, the involution $\pi_{0}$ of $S$ induces an automorphism (of Lie algebras) $\pi: \lambda \mapsto \pi_{0} \lambda \pi_{0}$ of $\mathfrak{o}(n)$ (the product $\pi_{0} \lambda \pi_{0}$ is taken in $\operatorname{End}_{F}(S)$ ). Since $\pi_{0}$ is an isometry of $(S, n), \pi_{0}$ induces an automorphism $C\left(\pi_{0}\right)$ of the Clifford algebra $C(S, n)$.

Lemma 45. For $\left(\begin{array}{ll}f & 0 \\ 0 & g\end{array}\right) \in \operatorname{End}_{F}(S \oplus S)$, we have

$$
\left(\alpha_{S} \circ C\left(\pi_{0}\right) \circ \alpha_{S}^{-1}\right)\left(\begin{array}{cc}
f & 0 \\
0 & g
\end{array}\right)=\left(\begin{array}{cc}
\pi(g) & 0 \\
0 & \pi(f)
\end{array}\right) .
$$

Proof. Since $C_{0}(S, n)$ is generated by all products $u v, u, v \in S$ (the product is taken in $C(S, n))$, it suffices to check the claim for $\left(\begin{array}{ll}f & 0 \\ 0 & g\end{array}\right)=\alpha_{S}(u v) \in \operatorname{End}_{F}(S \oplus S)$ which is a straightforward computation.

Theorem 46. If $S$ is of type $\mathrm{G}_{2}$ or is of type $\mathrm{A}_{2}$ for a split algebra, the relations $\pi^{2}=1$ and $\pi \circ \rho_{S}=\rho_{S}^{2} \circ \pi$ hold in $\operatorname{Aut}_{F}(\mathfrak{o}(n))$ and $\left\{\pi, \rho_{S}\right\}$ generate a subgroup of $\operatorname{Aut}_{F}(\mathfrak{o}(n))$ isomorphic to $S_{3}$.

Proof. The first relation is obvious. We check the second one. Since $\pi_{0}$ is an isometry of $(S, n), \pi_{0}$ induces an automorphism $C\left(\pi_{0}\right)$ of the Clifford algebra 
$C(S, n)$. We know that ado $C\left(\pi_{0}\right) \circ \operatorname{ad}^{-1}=\pi=\operatorname{Int}\left(\pi_{0}\right)$ on $\mathfrak{o}(n)$ by Proposition 39 ; further, $\left(\alpha_{S} \circ \operatorname{ad}^{-1}\right)(\lambda)=\left(\rho_{S}(\lambda), \rho_{S}^{2}(\lambda)\right)$, so that

$$
\begin{aligned}
\left(\pi\left(\rho_{S}^{2}(\lambda)\right), \pi\left(\rho_{S}(\lambda)\right)\right) & =\left(\alpha_{S} \circ C\left(\pi_{0}\right) \circ \alpha_{S}^{-1}\right)\left(\rho_{S}(\lambda), \rho_{S}^{2}(\lambda)\right) \\
& =\left(\alpha_{S} \circ C\left(\pi_{0}\right) \circ \operatorname{ad}^{-1}\right)(\lambda) \\
& =\left(\alpha_{S} \circ \operatorname{ad}^{-1} \circ \operatorname{ad} \circ C\left(\pi_{0}\right) \circ \operatorname{ad}^{-1}\right)(\lambda) \\
& =\left(\alpha_{S} \circ \operatorname{ad}^{-1}\right)(\pi(\lambda)) \\
& =\left(\rho_{S}(\pi(\lambda)), \rho_{S}^{2}(\pi(\lambda))\right) .
\end{aligned}
$$

The second relation then follows from Theorem 44. Thus we get get a homomorphism $S_{3} \rightarrow \operatorname{Aut}_{F}(\mathfrak{o}(n))$. It is obviously injective since $\rho_{S} \neq 1$.

Remark 47 . Let $(S, \star, n)$ be a symmetric composition and let $\left(\rho_{1}, \rho_{2}\right)$ be the two automorphisms of order 3 of the Lie algebra $\mathfrak{o}(n)$ constructed in Theorem 44 . If $(S, \diamond, n)$ is another symmetric composition with the same underlying quadratic space, we get a different pair of automorphisms $\left(\phi_{1}, \phi_{2}\right)$ of order 3 of the Lie algebra $\mathfrak{o}(n)$ and it follows from the exact sequence (2) that, at least over an algebraic closure of $F$ (and after renumbering $\phi_{1}, \phi_{2}$ if necessary), there exists $f, g \in \mathrm{GO}^{+}(n)$ such that $\left(\phi_{1}, \phi_{2}\right)=\operatorname{Int}(f, g)\left(\rho_{1}, \rho_{2}\right)$. This also follows directly from Theorem 43 and is valid over $F$. Let

$$
\alpha_{S}:\left(C_{0}(S, n), \tau\right) \stackrel{\sim}{\rightarrow}\left(\operatorname{End}_{F}(S), \sigma_{n}\right) \times\left(\operatorname{End}_{F}(S), \sigma_{n}\right)
$$

be the isomorphism defined in (41) and let $\alpha_{S}^{\prime}$ be the corresponding isomorphism defined via $\diamond$. Then $\alpha_{S}^{\prime} \circ \alpha_{S}^{-1}$ is an automorphism of $\left(\operatorname{End}_{F}(S), \sigma_{n}\right) \times\left(\operatorname{End}_{F}(S), \sigma_{n}\right)$ and we may assume that $\alpha_{S}^{\prime} \circ \alpha_{S}^{-1}=\operatorname{Int}(f, g)$ with $(f, g) \in \mathrm{GO}^{+}(n)$. Since $\left(\rho_{1}, \rho_{2}\right)=\alpha_{S} \circ \operatorname{ad}^{-1}$ and $\left(\phi_{1}, \phi_{2}\right)=\alpha_{S}^{\prime} \circ \operatorname{ad}^{-1}$ it follows that $\left(\alpha_{S}^{\prime} \circ \operatorname{ad}^{-1}\right) \circ\left(\right.$ ado $\alpha_{S}^{-1}=$ $\operatorname{Int}(f, g)$ and $\left(\phi_{1}, \phi_{2}\right)=\operatorname{Int}(f, g)\left(\rho_{1}, \rho_{2}\right)$.

\section{Automorphisms of order 3 and composition algebras}

A Lie algebra $\mathfrak{g}$ of type $\mathrm{D}_{4}$ over $F$ is a form of $\mathfrak{o}_{8}$, i.e., there exists an isomorphism

$$
\beta: \mathfrak{g} \otimes_{F} \bar{F} \stackrel{\sim}{\rightarrow} \overline{\mathfrak{o}}_{8}=\mathfrak{o}_{8} \otimes_{F} \bar{F},
$$

where $\bar{F}$ is an algebraic closure of $F$. The following result is well known. We include a proof via symmetric compositions for completeness.

Theorem 48. An orthogonal Lie algebra $\mathfrak{g}$ of type $\mathrm{D}_{4}$ over $F$ admits an outer automorphism of order 3 if and only if $\mathfrak{g}$ is isomorphic to the orthogonal Lie algebra of the norm form $n$ of a Cayley algebra $\mathcal{C}$ over $F$.

Proof. The fact that the orthogonal Lie algebra of the norm form $n$ of a Cayley algebra admits an outer automorphism of order 3 follows for example from Theorem 44. The proof of the converse relies on the following simple, but useful principle, see [KMRT, (2.25)]. 
Lemma 49. Let $\Omega$ and $C$ be $F$-algebras, and let $U \subset \Omega$ be a vector subspace which generates $\Omega$ as an F-algebra. Let $f: U \rightarrow C$ be an $F$-linear map. If there exists a field extension $K$ of $F$ such that $f \otimes_{F} K: U \otimes_{F} K \rightarrow C \otimes_{F} K$ extends to a $K$ algebra homomorphism $\hat{f}_{K}: \Omega \otimes_{F} K \rightarrow C \otimes_{F} K$, then $f$ extends to an $F$-algebra homomorphism $\hat{f}: \Omega \rightarrow C$.

Proof. The restriction of $\hat{f}_{K}$ to $\Omega$ is an $F$-algebra homomorphism $\Omega \rightarrow C \otimes_{F}$ $K$. Since the restriction of $\hat{f}_{K}$ to $U$ is $f$ and $U$ generates $\Omega$, the image of this homomorphism is in fact contained in $C$.

We return to the proof of Theorem 48 . Let $\bar{F}$ be an algebraic closure of $F$ and let $(\bar{V}, \bar{q})=(V, q) \otimes_{F} \bar{F}$. Then $(\bar{V}, \bar{q})$ is isometric to the norm of the split Cayley algebra $(\overline{\mathcal{C}}, \bar{n})$ since both quadratic forms are hyperbolic over $\bar{F}$. Let

$$
\beta:(\bar{V}, \bar{q}) \stackrel{\sim}{\rightarrow}(\overline{\mathcal{C}}, \bar{n})
$$

be an isometry. We have an induced commutative diagram

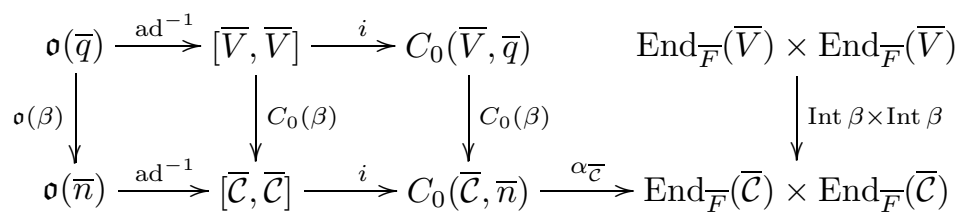

Let $\left(f_{1}^{\prime}, f_{2}^{\prime}\right)$ be the two components of the map $\mathfrak{o}(\bar{q})$ to $\mathfrak{o}(\bar{q}) \times \mathfrak{o}(\bar{q})$ given by the composition $(\operatorname{Int} \beta \times \operatorname{Int} \beta)^{-1} \circ \alpha_{\overline{\mathcal{C}}} \circ C_{0}(\beta) \circ i \circ \mathrm{ad}^{-1}$. By Theorem 43, $\left(f_{1}^{\prime}, f_{2}^{\prime}\right)$ is a pair of outer automorphisms of $\mathfrak{o}(\bar{q})$. Let $f_{1}$ be an outer automorphism of order 3 of $\mathfrak{o}(q)$ and let $f_{2}=f_{1}^{2}$. In view of Remark 47 (and renumbering $f_{1}$ and $f_{2}$ if necessary), there exist inner automorphisms Int $h_{1}$, Int $h_{2}$ of $\mathfrak{o}(\bar{q})$ such that

$$
f_{1}=\operatorname{Int} h_{1} \circ f_{1}^{\prime}, \quad f_{2}=\operatorname{Int} h_{2} \circ f_{2}^{\prime} .
$$

The composition

$$
\left(\operatorname{Int} h_{1} \times \operatorname{Int} h_{2}\right) \circ(\operatorname{Int} \beta \times \operatorname{Int} \beta)^{-1} \circ \alpha_{\overline{\mathcal{C}}} \circ C_{0}(\beta)
$$

maps the algebra $C_{0}(V, q)$ to $\operatorname{End}_{\bar{F}}(\bar{V}) \times \operatorname{End}_{\bar{F}}(\bar{V})$ and its restriction to $[V, V]$ has as image the image of $\left(f_{1}, f_{2}\right)$ which is contained in $\mathfrak{o}(q) \times \mathfrak{o}(q) \subset \operatorname{End}_{F}(V) \times \operatorname{End}_{F}(V)$. Since $\mathfrak{o}(q) \stackrel{\sim}{\rightarrow}[V, V]$ generates $C_{0}(V, q)$ as an algebra, it follows from Lemma 49 that $\left(f_{1}, f_{2}\right) \circ$ ad $^{-1}$ extends to isomorphism $\gamma: C_{0}(V, q) \stackrel{\sim}{\rightarrow} \operatorname{End}_{F}(V) \times \operatorname{End}_{F}(V)$. Replacing $q$ by a multiple $\lambda q$ if necessary, we may assume that there is an element $e \in V$ such that $q(e)=1$. Let

$$
V=F \cdot e \perp U
$$

For any $u \in U$, let $\gamma(e u)=\left(\phi_{u}, \psi_{u}\right) \in \operatorname{End}_{F}(V) \times \operatorname{End}_{F}(V)$. Since $e u+u e=0$ in $C_{0}(q)$, we have $2 e u=[e, u] \in[V, V]$ and $\left(\phi_{u}, \psi_{u}\right) \in \mathfrak{o}(q) \times \mathfrak{o}(q)$, moreover $\left(\phi_{u}, \psi_{u}\right)^{2}=\gamma(e u)^{2}=-q(u) \cdot(1,1)$ and

$$
b_{q}\left(\phi_{u}(y), \phi_{u}(z)\right)=-b_{q}\left(\phi_{u}^{2}(y), z\right)=q(u) b_{q}(y, z)
$$


for all $u \in U, y, z \in V$. Let $x=\lambda e+u \in V$. It follows from (51) that the bilinear product on $V$ given by

$$
x \diamond y=\lambda y+\phi_{u}(y), \quad y \in V
$$

satisfies

$$
b_{q}(x \diamond y, x \diamond y)=q(x) b_{q}(y, z),
$$

hence defines a composition algebra on $(V, q)$. By Proposition $9 q$ is the norm form of a Cayley algebra.

A result corresponding to Theorem 48 for projective groups of similitudes is proved in [WO] with a quite settled geometric argument.

Theorem 52. Let $\mathfrak{o}(n)$ be the orthogonal Lie algebra relative to a 3-Pfister form $n$.

(1) The cyclic subgroups $G(f)$ and $G(g)$ of Aut $(\mathfrak{o}(n))$ generated by two outer automorphisms $f, g$ of $\mathfrak{o}(n)$ of order 3 are conjugate and only if they have isomorphic fixed point subalgebras.

(2) Each conjugacy class of a cyclic subgroup $G(f)$ of Aut $(\mathfrak{o}(n))$, generated by an outer automorphism $f$ of order 3 , is the class of a subgroup $G\left(f_{S}\right)$ for an automorphism $f_{S}$ induced by some symmetric composition $(S, \star)$. The Lie algebra $\operatorname{Der}(S, \star)$ is the fixed point algebra of the group $G\left(f_{S}\right)$. Up to isomorphism the conjugacy class of $G\left(f_{S}\right)$ is uniquely determined by the $\operatorname{pair}\left\{S, S^{\mathrm{op}}\right\}$.

Proof. (1) If $G(f)$ and $G(g)$ are conjugate and for example $g=\phi \circ f \circ \phi^{-1}$ with $\phi \in \operatorname{Aut}(\mathfrak{o}(n))$, then $\phi$ restricts to an isomorphism $\operatorname{Fix}(G(f)) \stackrel{\sim}{\rightarrow} \operatorname{Fix}(G(g))$. Conversely, let $\mathfrak{g}=\operatorname{Fix}(G(f))$ and $\mathfrak{h}=\operatorname{Fix}(G(g))$ be isomorphic Lie algebras. By Theorem $6, \mathfrak{g}$ and $\mathfrak{h}$ are both of type $G_{2}$ or of type $A_{2}$. If they are both of type $\mathrm{G}_{2}$, they are isomorphic to derivation algebras of Cayley algebras $\mathcal{C}_{1}$ and $\mathcal{C}_{2}$. In view of Corollary 21 we may assume that $\mathcal{C}_{1}=\mathcal{C}_{2}=\mathcal{C}$, where $\mathcal{C}$ is the (up to isomorphism) unique Cayley algebra with norm $n$. Moreover, we can assume that $\operatorname{Der}(\mathcal{C})$ is canonically embedded in $\mathfrak{o}(n)$. Let $\phi_{1}: \mathfrak{g} \stackrel{\sim}{\rightarrow} \operatorname{Der}(\mathcal{C})$ and $\phi_{2}: \mathfrak{h} \stackrel{\sim}{\rightarrow} \operatorname{Der}(\mathcal{C})$ be isomorphisms. In view of Corollary 22, the isomorphisms $\phi_{1}$ and $\phi_{2}$ can be extended to inner automorphisms $s_{i}$ of $\mathfrak{o}(n)$. Conjugating $f$ by $s_{1}^{-1}$ and $g$ by $s_{2}^{-1}$ we are reduced to the case where $f$ and $g$ have the same fixed point algebra $\operatorname{Der}(\mathcal{C})$. It then follows from Remark 47 that $f \circ g^{-1}$ or $f^{2} \circ g^{-1}$ is an inner automorphism $s=\operatorname{Int}(t), t \in \mathrm{GO}^{+}(n)$. Let $\mathcal{C}=\mathcal{C}^{0} \perp F \cdot 1$. The image of $\operatorname{Der}(\mathcal{C})$ in $\mathfrak{o}(n) \subset \operatorname{End}_{F}(\mathcal{C})$ lies in $\operatorname{End}_{F}\left(\mathcal{C}^{0}\right)$ and $t$ commutes with this image. Thus $t$ is of the form $\mu \cdot 1_{\mathcal{C}^{0}}+\lambda \cdot 1_{F}, \lambda, \mu \in F^{\times}$. The multiplier of $t$ is equal to $\lambda^{2}$ and to $\mu^{2}$, so that $\lambda= \pm \mu$. Since $t$ is proper, the case $\lambda=-\mu$ cannot occur and $s=\operatorname{Int}(t)$ is the identity on $\mathfrak{o}(n)$. A similar proof, using Proposition 26 and Corollary 30, holds if both fixed point algebras are of type $A_{2}$. Observe that in this case, we only get conjugation up to automorphisms, not necessarily inner automorphisms.

(2) Let $f$ be an outer automorphism of order 3 of $\mathfrak{o}(n)$ and let $\operatorname{Fix}(f)=\mathfrak{g}$. Then $\mathfrak{g}$ is of type $\mathrm{G}_{2}$ or $A_{2}$. Assume first that $\mathfrak{g} \simeq \operatorname{Der}(\mathcal{C})$ for a Cayley algebra $\mathcal{C}$. Let $f_{\mathcal{C}}$ be an outer automorphism of $\mathfrak{o}(n)$ induced through the para-Cayley algebra $\mathcal{C}$ (see Theorem 44). In view of $(1) G(f)$ and $G\left(f_{\mathcal{C}}\right)$ are conjugate. If $\mathfrak{g}$ is of type $\mathrm{A}_{2}$ 
we know by descent that $\mathfrak{g} \simeq \operatorname{Skew}(B, \tau)^{0}$ for $B$ central simple of degree 3 over a quadratic extension $K / F$. In view of Corollary 30, $K$ must be isomorphic to $F[x] /\left(x^{2}+3\right)$. Let $(S, \star, n)$ be the symmetric composition associated with $(B, \tau)$. The fixed point algebra of the corresponding automorphism $f_{S}$ of order 3 of $\mathfrak{o}(n)$ is isomorphic to $\operatorname{Fix}(f)$. Hence by $(1)$ the cyclic subgroups $G(f)$ and $G\left(f_{S}\right)$ are in the same conjugacy class. Unicity follows from Proposition 35.

In view of the classification of symmetric compositions we have the following classification of outer automorphisms.

Corollary 53. Let $n$ be a 3-Pfister form over a field $F$ of characteristic zero and let $\operatorname{Conj}(n)$ be the set of conjugacy classes of order 3 outer automorphisms of the orthogonal algebra $\mathfrak{o}(n)$.

(1) If $F$ does not contain a primitive cubic root of 1 , then $\operatorname{Conj}(n)$ is in bijection with the set consisting of the isomorphism class of a Cayley algebra with norm $n$ and all $F(\sqrt{-3})$-isomorphism classes of pairs consisting of a central simple algebra of degree 3 over $F(\sqrt{-3}) / F$ and an involution of the second kind such that the cohomological invariant of the involution is the class of $n$.

(2) If $F$ contains a primitive cubic root of 1 and $n$ is hyperbolic, then $\operatorname{Conj}(n)$ is in bijection with the set consisting of the isomorphism class of the split Cayley algebra and the isomorphism classes of central simple algebras of degree 3 over $F$.

(3) If $F$ contains a primitive cubic root of 1 and $n$ is anisotropic, then $\operatorname{Conj}(n)$ reduces to the isomorphism class of a Cayley algebra with norm $n$.

Proof. (1) If the composition algebra $S$ admits an involution $\sigma_{S}$, then by Theorem $46 f_{S}$ and $f_{S}^{2}=f_{S \text { op }}$ are conjugate in $\operatorname{Aut}(\mathfrak{o}(n))$. Thus in this case the conjugacy class is determined by $S$. The left cases are where the central simple algebra $B$ of degree 3 is not split. Then $B$ and $B^{\text {op }}$ are not isomorphic so that $S$ and $S^{\text {op }}$ are not isomorphic either. The claim then follows from the fact that the form $n$ determines the isomorphism type of the involution of the second kind on $B$. Claims (2) and (3) follow from (1) and the fact that $n$ is hyperbolic if $F$ contains a primitive cubic root of unity (see Proposition 32).

Remark 54. As observed in [HKRT], given an algebra $B$ of degree 3 with an involution of the second kind and given a norm form $n, B$ does not necessarily admits an involution of the second kind with invariant $n$. However, since any central simple algebra $B$ of degree 3 with an involution of the second kind admits a distinguished involution, there is at least one class of outer automorphisms of order 3 for each isomorphism class of central simple algebras $B$ of degree 3 admitting an involution of the second kind over the extension $F[x] /\left(x^{2}+3\right)$.

Example 55. Over $\mathbb{R}$, there are two 3-Pfister forms, the hyperbolic form and the identity form, hence two isomorphism classes of orthogonal Lie algebras admitting outer automorphisms of order 3. For each one there is a conjugacy class of outer automorphisms of order 3 given by the corresponding Cayley algebra. Moreover, we have two classes of involutions of the second kind on $M_{3}(\mathbb{C}$ ) (see Example 34). For the Hermitian transpose $\tau$, the invariant is the identity form. 
The other class of involutions is given by the distinguished involution $\operatorname{Int}(u) \circ \tau$, where $u=\operatorname{diag}(-1,1,1)$ (see Example 34 ). Thus we have two classes of outer automorphisms of order 3 for each of the two 3-Pfister forms.

\section{Appendix (in collaboration with Larissa Cadorin)}

Using adequate multiplication tables of symmetric compositions, one can use Theorem 43 to give explicit descriptions of trialitarian actions defined through symmetric compositions. Computations of this kind were done in [KPS] and [C]. We first consider the case of a split para-Cayley algebra $\mathcal{C}_{s}$. Let $Q_{s}=M_{2}(F)$ be the split quaternion algebra, with basis $v_{1}=1, v_{2}, v_{3}, v_{4}=v_{2} v_{3}$ and relations $v_{2}^{2}=v_{3}^{2}=1$ and $v_{2} v_{3}+v_{3} v_{2}=0$. Let $x \mapsto \bar{x}$ be the conjugation in $Q_{s}$. We set $C_{s}=Q_{s}+v_{5} Q_{s}$ and define the multiplication in $\mathcal{C}_{s}$ by

$$
\left(a+v_{5} b\right)\left(a^{\prime}+v_{5} b^{\prime}\right)=\left(a a^{\prime}+b^{\prime} \bar{b}\right)+v_{5}\left(\bar{a} b^{\prime}+a^{\prime} b\right) \quad \text { for } a, a^{\prime}, b, b^{\prime} \in Q_{s} .
$$

The norm $n$ of $\mathcal{C}_{s}$ with respect to the basis $\left(v_{1}, \ldots, v_{4}, v_{5}, v_{6}=v_{5} v_{2}, v_{7}=v_{5} v_{3}, v_{8}=\right.$ $\left.v_{5} v_{7}\right)$ is the diagonal form $\langle 1,-1, \ldots, 1,-1\rangle$. It is more convenient to work with the basis

$$
\begin{array}{llll}
e_{1}=\frac{1}{2}\left(v_{4}-v_{8}\right), & e_{2}=\frac{1}{2}\left(-v_{2}+v_{6}\right), & e_{3}=\frac{1}{2}\left(-v_{3}+v_{7}\right), & e_{4}=\frac{1}{2}\left(v_{1}+v_{5}\right), \\
f_{1}=\frac{1}{2}\left(v_{4}+v_{8}\right), & f_{2}=\frac{1}{2}\left(v_{2}+v_{6}\right), & f_{3}=\frac{1}{2}\left(v_{3}+v_{7}\right), & f_{4}=\frac{1}{2}\left(v_{1}-v_{5}\right),
\end{array}
$$

which is hyperbolic for $n$. The multiplication table for the corresponding paraCayley algebra is given in Table 1 .

TABLE 1.

\begin{tabular}{c|cc|cc|cc|cc}
\hline$\star$ & $e_{1}$ & $f_{1}$ & $e_{2}$ & $f_{2}$ & $e_{3}$ & $f_{3}$ & $e_{4}$ & $f_{4}$ \\
\hline$e_{1}$ & 0 & $-f_{4}$ & $f_{3}$ & 0 & $-f_{2}$ & 0 & 0 & $-e_{1}$ \\
$f_{1}$ & $-e_{4}$ & 0 & 0 & $e_{3}$ & 0 & $-e_{2}$ & $-f_{1}$ & 0 \\
\hline$e_{2}$ & $-f_{3}$ & 0 & 0 & $-f_{4}$ & $f_{1}$ & 0 & 0 & $-e_{2}$ \\
$f_{2}$ & 0 & $-e_{3}$ & $-e_{4}$ & 0 & 0 & $e_{1}$ & $-f_{2}$ & 0 \\
\hline$e_{3}$ & $f_{2}$ & 0 & $-f_{1}$ & 0 & 0 & $-f_{4}$ & 0 & $-e_{3}$ \\
$f_{3}$ & 0 & $e_{2}$ & 0 & $-e_{1}$ & $-e_{4}$ & 0 & $-f_{3}$ & 0 \\
\hline$e_{4}$ & $-e_{1}$ & 0 & $-e_{2}$ & 0 & $-e_{3}$ & 0 & $f_{4}$ & 0 \\
$f_{4}$ & 0 & $-f_{1}$ & 0 & $-f_{2}$ & 0 & $-f_{3}$ & 0 & $e_{4}$ \\
\hline
\end{tabular}

We have $1=e_{4}+f_{4}$ and conjugation in $\mathcal{C}_{s}$ is given by $\pi\left(e_{i}\right)=-e_{i}, \pi\left(f_{i}\right)=-f_{i}$ for $i=1, \ldots, 3$ and $\pi\left(e_{4}\right)=f_{4}$. The following relations hold in the Clifford algebra $C\left(\mathcal{C}_{s}, n\right)$ :

$$
e_{i} e_{j}+e_{j} e_{i}=f_{i} f_{j}+f_{j} f_{i}=0 \quad \text { and } \quad e_{i} f_{j}+f_{j} e_{i}=\delta_{i j}
$$

for all $i, j=1, \ldots, 4$. A computation shows that the isomorphism $\operatorname{ad}^{-1}: \mathfrak{o}_{8} \stackrel{\sim}{\rightarrow}$ 
$\left[\mathcal{C}_{s}, \mathcal{C}_{s}\right] \subset C_{0}\left(\mathcal{C}_{s}, n\right)$ of Lemma 38 is given by

$$
\begin{aligned}
& G_{i j}^{Y}=E_{i, j+4}-E_{j, i+4} \quad \mapsto e_{i} e_{j}, \quad 1 \leqslant i<j \leqslant 4 \\
& G_{i j}^{Z}=E_{i+4, j}-E_{j+4, i+4} \mapsto f_{i} f_{j}, \quad 1 \leqslant i<j \leqslant 4, \\
& G_{i j}^{X}=E_{i, j}-E_{j+4, i+4} \quad \mapsto e_{i} f_{j}, \quad 1 \leqslant i, j \leqslant 4, i \neq j, \\
& G_{i i}^{X}=E_{i, i}-E_{i+4, i+4} \quad \mapsto e_{i} f_{i}-\frac{1}{2}, \quad 1 \leqslant i \leqslant 4 .
\end{aligned}
$$

The image of a generic skew-symmetric matrix

$$
\begin{aligned}
\mathcal{X} & =\sum_{1 \leqslant i, j \leqslant 4} x_{i j} G_{i j}^{X}+\sum_{1 \leqslant i<j \leqslant 4} y_{i j} G_{i j}^{Y}+\sum_{1 \leqslant i<j \leqslant 4} z_{i j} G_{i j}^{Z} \\
& =\left(\begin{array}{cccc|cccc}
x_{11} & x_{12} & x_{13} & x_{14} & 0 & y_{12} & y_{13} & y_{14} \\
x_{21} & x_{22} & x_{23} & x_{24} & -y_{12} & 0 & y_{23} & y_{24} \\
x_{31} & x_{32} & x_{33} & x_{34} & -y_{13} & -y_{23} & 0 & y_{34} \\
x_{41} & x_{42} & x_{43} & x_{44} & -y_{14} & -y_{24} & -y_{34} & 0 \\
\hline 0 & z_{12} & z_{13} & z_{14} & -x_{11} & -x_{21} & -x_{31} & -x_{41} \\
-z_{12} & 0 & z_{23} & z_{24} & -x_{12} & -x_{22} & -x_{32} & -x_{42} \\
-z_{13} & -z_{23} & 0 & z_{34} & -x_{13} & -x_{23} & -x_{33} & -x_{43} \\
-z_{14} & -z_{24} & -z_{34} & 0 & -x_{14} & -x_{24} & -x_{34} & -x_{44}
\end{array}\right)
\end{aligned}
$$

under the map $\alpha_{\mathcal{C}_{s}} \circ \mathrm{ad}^{-1}$ of (42) is

$$
\begin{aligned}
\alpha_{S} \circ \operatorname{ad}^{-1}(\mathcal{X}) & \\
= & \sum_{\substack{1 \leqslant i, j \leqslant 4 \\
i \neq j}} x_{i j}\left(\begin{array}{cc}
L_{e_{i}} R_{f_{j}} & 0 \\
0 & R_{e_{i}} L_{f_{j}}
\end{array}\right)+\sum_{i=1}^{4} x_{i i}\left[\left(\begin{array}{cc}
L_{e_{i}} R_{f_{i}} & 0 \\
0 & R_{e_{i}} L_{f_{i}}
\end{array}\right)-\frac{1}{2}\left(\begin{array}{ll}
I & 0 \\
0 & I
\end{array}\right)\right] \\
& +\sum_{1 \leqslant i<j \leqslant 4} y_{i j}\left(\begin{array}{cc}
L_{e_{i}} R_{e_{j}} & 0 \\
0 & R_{e_{i}} L_{e_{j}}
\end{array}\right)+\sum_{1 \leqslant i<j \leqslant 4} z_{i j}\left(\begin{array}{cc}
L_{f_{i}} R_{f_{j}} & 0 \\
0 & R_{f_{i}} L_{f_{j}}
\end{array}\right)
\end{aligned}
$$

Let $\alpha_{\mathcal{C}_{s}} \circ \operatorname{ad}^{-1}\left(\mathcal{C}_{s}\right)=\left(\rho(\mathcal{X}), \rho^{2}(\mathcal{X})\right)$. Using the multiplication table (Table 1$)$, it is straightforward to show that $\rho(\mathcal{X})$ is given by

$$
\left(\begin{array}{cccc|cccc}
+--+ & x_{12} & x_{13} & -y_{14} & 0 & -x_{43} & x_{42} & -z_{23} \\
x_{21} & -+-+ & x_{23} & -y_{24} & x_{43} & 0 & -x_{41} & z_{13} \\
x_{31} & x_{32} & --++ & -y_{34} & -x_{42} & x_{41} & 0 & -z_{12} \\
z_{14} & z_{24} & z_{34} & ---- & z_{23} & -z_{13} & z_{12} & 0 \\
\hline 0 & x_{34} & -x_{24} & -y_{23} & -++- & -x_{21} & -x_{31} & -z_{14} \\
-x_{34} & 0 & x_{14} & y_{13} & -x_{12} & +-+- & -x_{32} & -z_{24} \\
x_{24} & -x_{14} & 0 & -y_{12} & -x_{13} & -x_{23} & ++-- & -z_{34} \\
y_{23} & -y_{13} & y_{12} & 0 & y_{14} & y_{24} & y_{34} & ++++
\end{array}\right)
$$


and $\rho^{2}(\mathcal{X})$ by

$$
\left(\begin{array}{cccc|cccc}
+--- & x_{12} & x_{13} & z_{23} & 0 & -z_{34} & z_{24} & -x_{14} \\
x_{21} & -+-- & x_{23} & -z_{13} & z_{34} & 0 & -z_{14} & -x_{24} \\
x_{31} & x_{32} & --+- & z_{12} & -z_{24} & z_{14} & 0 & -x_{34} \\
-y_{23} & y_{13} & -y_{12} & +++- & x_{14} & x_{24} & x_{34} & 0 \\
\hline 0 & -y_{34} & y_{24} & x_{41} & -+++ & -x_{21} & -x_{31} & y_{23} \\
y_{34} & 0 & -y_{14} & x_{42} & -x_{12} & +-++ & -x_{32} & -y_{13} \\
-y_{24} & y_{14} & 0 & x_{43} & -x_{13} & -x_{23} & ++-+ & y_{12} \\
-x_{41} & -x_{42} & -x_{43} & 0 & -z_{23} & z_{13} & -z_{12} & ---+
\end{array}\right)
$$

where $+/-$ are the signs of $\frac{1}{2}\left(x_{11}+x_{22}+x_{33}+x_{44}\right)$.

The automorphism $\rho$ restricts to an automorphism of the Cartan subalgebra $\mathfrak{h}$ which we used already in Section 2; the action is given by the matrix

$$
X=\frac{1}{2}\left(\begin{array}{cccc}
1 & -1 & -1 & 1 \\
-1 & 1 & -1 & 1 \\
-1 & -1 & 1 & 1 \\
-1 & -1 & -1 & -1
\end{array}\right)
$$

with respect to the basis $u_{j}=E_{j j}-E_{j+4, j+4}, j=1, \ldots, 4$ of $\mathfrak{h}$. We recall (see (5)) that the matrix $P$ of the automorphism $f_{\rho}$ induced on $\mathfrak{h}$ by the corresponding automorphism of the Dynkin diagram is

$$
R=\frac{1}{2}\left(\begin{array}{cccc}
1 & 1 & 1 & -1 \\
1 & 1 & -1 & 1 \\
1 & -1 & 1 & 1 \\
1 & -1 & -1 & -1
\end{array}\right)
$$

Obviously we have $X=Y R Y^{-1}$ with $Y=\operatorname{diag}(-1,1,1,1)$. This shows explicitly that both actions $\rho$ and $f_{\rho}$ are conjugate.

Similar computations can be made for all actions constructed with symmetric compositions. We sketch the case of the split algebra of type $A_{2}$. We represent $M_{3}(F)$ as a cyclic algebra with generators $x, y$ and relations

$$
y x=\omega x y, \quad x^{3}=1, \quad y^{3}=1,
$$

where $\omega$ is a primitive cubic root of 1 . The monomials $x^{i} y^{j},(i, j) \neq(0,0)>$ $0, i, j \leqslant 2$ have trace 0 and form a basis of $M_{3}(F)^{0}$. The basis

$$
\begin{array}{llll}
e_{1}=-x, & e_{2}=-y, & e_{3}=-\omega x y, & e_{4}=-\omega^{2} x^{2} y, \\
f_{1}=-x^{2}, & f_{2}=-y^{2}, & f_{3}=-\omega x^{2} y^{2}, & f_{4}=-\omega^{2} y x^{2},
\end{array}
$$

is hyperbolic and the multiplication $\star$ has the following nice multiplication table (Table 2)(see $[\mathrm{E}])$. 
TABle 2.

\begin{tabular}{c|cc|cc|cc|cc}
\hline$\star$ & $e_{1}$ & $f_{1}$ & $e_{2}$ & $f_{2}$ & $e_{3}$ & $f_{3}$ & $e_{4}$ & $f_{4}$ \\
\hline$e_{1}$ & $-f_{1}$ & 0 & 0 & $f_{4}$ & 0 & $f_{2}$ & 0 & $f_{3}$ \\
$f_{1}$ & 0 & $-e_{1}$ & $e_{4}$ & 0 & $e_{2}$ & 0 & $e_{3}$ & 0 \\
\hline$e_{2}$ & $e_{3}$ & 0 & $-f_{2}$ & 0 & $f_{4}$ & 0 & 0 & $e_{1}$ \\
$f_{2}$ & 0 & $f_{3}$ & 0 & $-e_{2}$ & 0 & $e_{4}$ & $f_{1}$ & 0 \\
\hline$e_{3}$ & $e_{4}$ & 0 & 0 & $e_{1}$ & $-f_{3}$ & 0 & $f_{2}$ & 0 \\
$f_{3}$ & 0 & $f_{4}$ & $f_{1}$ & 0 & 0 & $-e_{3}$ & 0 & $e_{2}$ \\
\hline$e_{4}$ & $e_{2}$ & 0 & $f_{3}$ & 0 & 0 & $e_{1}$ & $-f_{4}$ & 0 \\
$f_{4}$ & 0 & $f_{2}$ & 0 & $e_{3}$ & $f_{1}$ & 0 & 0 & $-e_{4}$ \\
\hline
\end{tabular}

Since the norm form is hyperbolic we choose the same Cartan subalgebra $\mathfrak{H}$ of $\mathfrak{o}_{8}$ as above and similar computations give a trialitarian action $\rho^{\prime}$. The image of the generic skew-symmetric matrix (57) under $\rho^{\prime}$ is

$$
\left(\begin{array}{cccc|cccc}
-+++ & y_{23} & y_{34} & -y_{24} & 0 & x_{41} & x_{21} & x_{31} \\
-z_{23} & ---+ & x_{42} & x_{43} & -x_{41} & 0 & -z_{13} & z_{12} \\
-z_{34} & x_{24} & -+-- & x_{23} & -x_{21} & z_{13} & 0 & -z_{14} \\
z_{24} & x_{34} & x_{32} & --+- & -x_{31} & -z_{12} & z_{14} & 0 \\
\hline 0 & -x_{14} & -x_{12} & -x_{13} & +--- & z_{23} & -z_{34} & -z_{24} \\
x_{14} & 0 & -y_{13} & y_{12} & -y_{23} & +++- & -x_{24} & -x_{34} \\
x_{12} & y_{13} & 0 & -y_{14} & -y_{34} & -x_{42} & +-++ & -x_{32} \\
x_{13} & -y_{12} & y_{14} & 0 & y_{24} & -x_{43} & -x_{23} & ++-+
\end{array}\right),
$$

where $+/-$ are as above the signs of $\frac{1}{2}\left(x_{11}+x_{22}+x_{33}+x_{44}\right)$. Here again the action restricts to an automorphism of the Cartan algebra, given by the matrix

$$
X^{\prime}=\frac{1}{2}\left(\begin{array}{cccc}
-1 & 1 & 1 & 1 \\
-1 & -1 & -1 & 1 \\
-1 & 1 & -1 & -1 \\
-1 & -1 & +1 & -1
\end{array}\right)
$$

Since $\operatorname{tr}\left(X^{\prime}\right)=-2$ and $\operatorname{tr}(X)=1, X$ and $X^{\prime}$ are clearly not conjugate.

\section{References}

[C] L. Cadorin, Triality, Master's thesis, ETH Zürich, 2000.

[E] A. Elduque, Symmetric composition algebras, J. Algebra 196 (1997), 283-300.

[EM] A. Elduque, H. C. Myung, On flexible composition algebras, Comm. Algebra 21 (1993), 2481-2505.

[F] J. R. Faulkner, Finding octonion algebras in associative algebras, Proc. Amer. Math. Soc. 104 (1988), 1027-1030. 
[H] S. Helgason, Differential Geometry, Lie Groups, and Symmetric Spaces, Pure and Applied Mathematics, Vol. 80, Academic Press, New York, 1978.

[HKRT] D. E. Haile, M.-A. Knus, M. Rost, J.-P. Tignol, Algebras of odd degree with involution, trace forms and dihedral extensions, Israel J. Math. 96 (1996), 299340 .

[J39] N. Jacobson, Cayley numbers and simple Lie algebras of type G, Duke Math. J. 5 (1939), 775-783.

[J62] N. Jacobson, Lie Algebras, Dover, New York, 1979, Republication of the 1962 original.

[J64] N. Jacobson, Triality and Lie algebras of type $D_{4}$, Rend. Circ. Mat. Palermo (2) 13 (1964), 129-153.

[JZ] Q. Jin, Z. Zhang, On automorphisms of affine Kac-Moody algebras, Comm. Algebra 29 (2001), 2827-2858.

[K69] В. Г. Кац, Автоморфизмы конечного порядка полупростых алгебр Ли, Функц. анал. и его прилож. 3 (1969), nо. 3, 94-96. English transl.: V. G. Kac, Automorphisms of finite order of semisimple Lie algebras, Funct. Anal. Appl. 3 (1969), 252-254.

[K85] V. G. Kac, Infinite-dimensional Lie Algebras, Cambridge University Press, Cambridge, 1985. Russian transl.: В. Г. Кац, Бесконечномерные алгебры Ли, Мир, М., 1993.

[KA] I. Kaplansky, Infinite-dimensional quadratic forms admitting composition, Proc. Amer. Math. Soc. 4 (1953), 956-960.

[KMRT] M.-A. Knus, A. Merkurjev, M. Rost, J.-P. Tignol, The Book of Involutions, American Mathematical Society Colloquium Publications, Vol. 44, American Mathematical Society, Providence, RI, 1998.

[KPS] M.-A. Knus, R. Parimala, R. Sridharan, On generic triality, in: Algebra, Arithmetic and Geometry, Parts I, II (Mumbai, 2000), Tata Inst. Fund. Res. Stud. Math., Vol. 16, Tata Inst. Fund. Res., Bombay, 2002, pp. 355-404.

[N] E. Neher, Lie algebra automorphisms of finite order, preprint, 8 pp., 1979.

[O] S. Okubo, Pseudo-quaternion and pseudo-octonion algebras, Hadronic J. 1 (1978) 1250-1278.

[OO] S. Okubo, J. M. Osborn, Algebras with nondegenerate associative symmetric bilinear forms permitting composition, Comm. Algebra 9 (1981), 1233-1261.

[S] R. D. Schafer, An Introduction to Nonassociative Algebras, Pure and Applied Mathematics, Vol. 22, Academic Press, New York, 1966.

[SCH] W. Scharlau, Quadratic and Hermitian Forms, Grundlehren der mathematischen Wissenschaften, Vol. 270, Springer-Verlag, Berlin, 1985.

[SP] T. A. Springer, Linear Algebraic Groups, 2nd ed., Progress in Mathematics, Vol. 9, Birkhäuser, Boston, MA, 1998.

[SV] T. A. Springer, F. D. Veldkamp, Octonions, Jordan Algebras and Exceptional Groups, Springer Monographs in Mathematics, Springer-Verlag, Berlin, 2000.

[T] J. Tits, Sur la trialité et les algèbres d'octaves, Acad. Roy. Belg. Bull. Cl. Sci. (5) 44 (1958), 332-350.

[VBS] F. van der Blij, T. A. Springer, Octaves and triality, Nieuw Arch. Wiskd. (3) 8 (1960), 158-169. 
[WG] J. A. Wolf, A. Gray, Homogeneous spaces defined by Lie group automorphisms, J. Differential Geom. 2 (1968), 77-114.

[WO] M. J. Wonenburger, The automorphisms of $\mathrm{PO}_{8}^{+}(Q)$ and $\operatorname{PS}_{8}^{+}(Q)$, Amer. J. Math. 84 (1962), 635-641. 